\title{
Difference between Semicircular-like Laws Induced by p-Adic Number Fields and the Semicircular Law
}

\author{
Ilwoo Cho* \\ Department of Mathematics \& Statistics, Saint Ambrose University, \\ Davenport, Iowa, U. S. A. \\ *Corresponding author: choilwoo@sau.edu
}

Received February 02, 2017; Revised March 07, 2017; Accepted August 16, 2017

\begin{abstract}
In this paper, we study "semicircular-like" elements in free product Banach *-algebras induced by Haar-measurable functions over $p$-adic number fields $\mathbb{Q}_{p}$, for primes $p$. And we investigate how the free distributions of operators generated by our mutually-free weighted-semicircular elements are close enough to (or far from) those of free reduced words generated by arbitrary mutually-free semicircular elements.
\end{abstract}

Keywords: free probability, p-Adic number fields $\mathbb{Q}_{p}$, Hilbert-space representations, weighted-semicircular elements, semicircular elements, weight ratios

Cite This Article: Ilwoo Cho, "Difference between Semicircular-like Laws Induced by $p$-Adic Number Fields and the Semicircular Law.” Turkish Journal of Analysis and Number Theory, vol. 5, no. 5 (2017): 165-190. doi: 10.12691/tjant-5-5-4.

\section{Introduction}

The main purposes of this paper are (i) to establish weighted-semicircular elements and corresponding semicircular elements in a certain Banach *-probability space induced by measurable functions over p-adic number fields $\mathbb{Q}_{p}$, for primes $p$, (ii) to consider free-distributional data of weighted-semicircular elements, and those of operators generated by free, weighted-semicircular elements under free product, and (iii) to investigate how the free-distributional data of the operators generated by weighted-semicircular elements are close to those of operators generated by semicircular elements. In particular, the main results of the topic (iii) illustrate how the weights of our weighted-semicircular elements distort (or affect) the semicircular law, and such distortions are measured by so-called the weight-ratios of weighted-semicircular elements.

\subsection{Preview and Motivation}

We have considered how primes act on operator algebras. The relations between primes and operators have been studied in various different approaches. For instance, we studied how primes act on certain von Neumann algebras generated by $p$-adic, and Adelic measure spaces (e.g., [6]). Meanwhile, in [5], primes are regarded as linear functionals acting on arithmetic functions. In such a case, one can understand arithmetic functions as Krein-space operators (for fixed primes), under Krein-space representations (e.g., [8]). Also, in [1],
[3], [4] and [7], we considered free-probabilistic structures on a Hecke algebra $\mathcal{H}\left(G L_{2}\left(\mathbb{Q}_{p}\right)\right)$ for a fixed prime $p$.

In [2], we considered certain free random variables in a *-algebra $\mathcal{M}_{p}$, consisting of all measurable functions over $\mathbb{Q}_{p}$, for primes $p$, and its Hilbert-space representation. Under representation, corresponding $C^{*}$-algebras $M_{p}$ of $\mathcal{M}_{p}$ are constructed, and free probability on $M_{p}$ is studied there. In particular, for all $j \in \mathbb{Z}$, we define $C^{*}$-probability spaces $\left(M_{p}, \varphi_{j}^{p}\right)$, where $\varphi_{j}^{p}$ are kind of sectionized linear functional implying the number-theoretic free-distributional data on $\mathcal{M}_{p}$, in terms of the usual p-adic integration on $\mathbb{Q}_{p}$. Moreover, from the system

$$
\left\{\left(M_{p}, \varphi_{j}^{p}\right): p \in \mathcal{P}, j \in \mathbb{Z}\right\},
$$

of $C^{*}$-probability spaces, we constructed, and studied free probability on the free product $C^{*}$-probability space,

$$
\left(M_{\mathcal{P}}(\mathbb{Z}), \varphi\right)=\underset{p \in \mathcal{P}, j \in \mathbb{Z}}{\star}\left(M_{p}, \varphi_{j}^{p}\right),
$$

called the Adelic $C^{*}$-probability space.

Motivated by the main results of [2], in the paper [4], by using the free-probabilistic information from a single $C^{*}$-probability space $\left(M_{p}, \varphi_{j}^{p}\right)$, we established weighted-semicircular elements in a free product Banach 
* -probability space generated by $M_{p}$, and realized that these operators generate semicircular elements.

\subsection{Overview}

In Sections 2, we briefly introduce backgrounds and a motivation of our proceeding works.

Our free-probabilistic models on $\mathcal{M}_{p}$ is established and considered in Sections 3. And then, in Section 4, we construct suitable Hilbert-space representations of the free-probabilistic models of Section 3, preserving the freedistributional data implying number-theoretic information. Under representation, corresponding $C^{*}$-algebras $M_{p}$ are constructed from $\mathcal{M}_{p}$.

In Section 5, we consider $C^{*}$-subalgebraic structures $\mathcal{S}_{p}$ in $M_{p}$, generated by mutually-orthogonal $|\mathbb{Z}|$-many projections, sectionizing or filterizing $M_{p}$, and study free probability on them, for primes $p$.

In Sections 6, we construct certain Banach *-probability spaces $\mathfrak{L S}_{p}$ by defining so-called the radial operators $l_{p}$ on $\mathfrak{S}_{p}$. And we realize that the generating operators of $\mathfrak{L S}_{p}$ are weighted-semicircular under suitable linear functionals on $\mathfrak{L S}_{p}$, for every prime $p$. These weighted-semicircular elements generate semicircular elements in $\mathfrak{L S}_{p}$.

In Section 7, we enlarge our weighted-semicircularity and semicircularity under free product Banach *-probability space $\mathfrak{L} \mathfrak{S}$ of $\mathfrak{L S}_{p}$ 's. And, free distributions of operators generated by free weighted-semicircular family, and those of operators generated by free semicircular family are computed, in Sections 8, 9 and 10.

In Section 11, the close-ness (or difference) between free distributions of self-adjoint operators generated by semicircular elements, and those of operators generated by our weighted-semicircular elements is considered by defining so-called the weight-ratios. Such weight-ratios measure how our weighted-semicircularity is close to semicircularity.

\section{Preliminaries}

In this section, we briefly mention about backgrounds of our proceeding works.

\subsection{Free Probability}

Readers can check fundamental analytic-and-combinatorial free probability from [12] and [13] (and the cited papers therein). Free probability is understood as the non-commutative operator-algebraic version of classical probability theory (covering commutative cases). The classical independence is replaced by the freeness, by replacing measures to linear functionals. It has various applications not only in pure mathematics (e.g., [11]), but also in related scientific topics (for example, see $[3,4,6,8]$ ).
In particular, we will use combinatorial approach of Speicher (e.g., [12]).

Especially, in the text, without introducing detailed definitions and combinatorial backgrounds, free moments and free cumulants of operators will be computed. Also, we use free product of $C^{*}$-probability spaces in the sense of [12] and [13], without detailed introduction. However, rough introduction would be given whenever they are needed in text.

\subsection{Calculus on $\mathbb{Q}_{p}$}

For more about $p$-adic, or Adelic analysis, see [13]. Let $\mathbb{Q}_{p}$ be the $p$-adic number fields for $p \in \mathcal{P}$, equipped with the non-Archimedean $p$-norms $\|_{p}$ (on $\mathbb{Q}$ ), where $\mathcal{P}$ is the set of all primes in the natural numbers (or the positive integers) $\mathbb{N}$. This Banach space $\mathbb{Q}_{p}$ is also understood as a measure space

$$
\mathbb{Q}_{p}=\left(\mathbb{Q}_{p}, \sigma\left(\mathbb{Q}_{p}\right), \mu_{p}\right),
$$

equipped with the left-and-right additive invariant Haar measure $\mu_{p}$ on the $\sigma$-algebra $\sigma\left(\mathbb{Q}_{p}\right)$. Recall also that, $\mathbb{Q}_{p}$ is a well-defined ring algebraically. If $x \in \mathbb{Q}_{p}$, then

$$
x=\sum_{n=-N}^{\infty} x_{n} p^{n} \text { with } x_{n} \in\{0,1, \ldots, p-1\}
$$

for some $N \in \mathbb{N}$, i.e.,

$$
x=\left(\sum_{k=-N}^{-1} x_{k} p^{k}\right)+\left(\sum_{n=0}^{\infty} x_{n} p^{n}\right) \text { in } \mathbb{Q}_{p} .
$$

If $N \geq 0$, and hence, if $x=\sum_{n=0}^{\infty} x_{n} p^{n}$ in $\mathbb{Q}_{p}$, then $x$ is said to be a $p$-adic integer of $\mathbb{Q}_{p}$. The subset $\mathbb{Z}_{p}$ of $\mathbb{Q}_{p}$, consisting of all $p$-adic integers, is called the unit disk of $\mathbb{Q}_{p}$. Indeed, every $p$-adic integer $x$ satisfies $|x|_{p} \leq 1$. i.e.,

$$
\mathbb{Z}_{p}=\left\{x \in \mathbb{Q}_{p}:|x|_{p} \leq 1\right\}
$$

Remark that $\mu_{p}\left(\mathbb{Z}_{p}\right)=1$, and all $\mu_{p}$-measurable subsets $Y \in \sigma\left(\mathbb{Q}_{p}\right)$ are either finite-or-infinite unions, or finite intersections of the subsets formed by

$$
y+z \mathbb{Z}_{p}, \text { for } y, z \in \mathbb{Q}_{p} .
$$

As a topological space, the $p$-adic number field $\mathbb{Q}_{p}$ contains its basis elements

$$
U_{k}=p^{k} \mathbb{Z}_{p}, \text { for all } k \in \mathbb{Z},
$$

satisfying the basis property, 


$$
\mathbb{Q}_{p}=\bigcup_{k \in \mathbb{Z}} U_{k}
$$

and the chain property,

$$
\ldots \subset U_{2} \subset U_{1} \subset U_{0}=\mathbb{Z}_{p} \subset U_{-1} \subset U_{-2} \subset \ldots,
$$

and the measure-theoretic data,

$$
\mu_{p}\left(U_{k}\right)=\frac{1}{p^{k}}=\mu_{p}\left(x+U_{k}\right), \text { for all } k \in \mathbb{Z},
$$

for all $x \in \mathbb{Q}_{p}$.

By understanding $\mathbb{Q}_{p}$ as a measure space, one can establish a *-algebra $\mathcal{M}_{p}$ over $\mathbb{C}$ as a *-algebra consisting of all $\mu_{p}$-measurable functions $f$,

$$
f=\sum_{S \in \sigma\left(\mathbb{Q}_{p}\right)} t_{S} \chi_{S} \text { with } t_{S} \in \mathbb{C},
$$

where the sum $\Sigma$ means a finite sum, and $\chi_{S}$ are the usual characteristic functions of $S$. Of course, the adjoint $f^{*}$ of $f$ is defined to be

$$
f^{*}=\sum_{S \in \sigma\left(\mathbb{Q}_{p}\right)} \overline{t_{S}} \chi_{S} \text { in } \mathcal{M}_{p},
$$

where $\bar{z}$ mean the conjugates of $z$, for all $z \in \mathbb{C}$.

On $\mathcal{M}_{p}$, one can naturally define a linear functional $\varphi_{p}$,

$$
\varphi_{p}(f)=\int_{\mathbb{Q}_{p}} f d \mu_{p} \text {, for all } f \in \mathcal{M}_{p},
$$

and hence, the pair $\left(\mathcal{M}_{p}, \varphi_{p}\right)$ forms a well-determined *-probability space.

Remark 2.1. Remark that our *-probabilistic structure is a "commutative" measure-theoretic structure which is not under usual free-probability-theory point of view. However, free probability theory naturally covers measure theory on commutative algebras, and hence, we use the terminology, “*-probability space," under enlarged sense in this text.

Define now subsets $\partial_{k}$ of $\mathbb{Q}_{p}$ by

$$
\partial_{k}=U_{k} \backslash U_{k+1} \text {, for all } k \in \mathbb{Z} \text {. }
$$

We call such $\mu_{p}$-measurable subsets $\partial_{k}$, the $k$-th boundaries of the basis elements $U_{k}$ of (2.2.1), which are also $\mu_{p}$-measurable subsets, for all $k \in \mathbb{Z}$. By the basis property in (2.2.1), one obtains that

$$
\mathbb{Q}_{p}=\underset{k \in \mathbb{Z}}{\sqcup} \partial_{k},
$$

where $\sqcup$ means the disjoint union. Also, by measure-theoretic data in (2.2.1), one has

$$
\mu_{p}\left(\partial_{k}\right)=\mu_{p}\left(U_{k}\right)-\mu_{p}\left(U_{k+1}\right)=\frac{1}{p^{k}}-\frac{1}{p^{k+1}},
$$

for all $k \in \mathbb{Z}$.
Now, let $\mathcal{M}_{p}$ be as above, and let $f \in \mathcal{M}_{p}$. Then, by definition, $f$ is expressed by

$$
f=\sum_{S \in \sigma\left(\mathbb{Q}_{p}\right)} t_{S} \chi_{S} \text { with } t_{S} \in \mathbb{C},
$$

where $\mathrm{P}$ means the finite sum, and $\chi_{S}$ are the usual characteristic functions of $S \in \sigma\left(\mathbb{Q}_{p}\right)$.

If $f \in \mathcal{M}_{p}$ is expressed by (2.2.5), then the adjoint $f^{*}$ is determined to be

$$
\left(\sum_{S \in \sigma\left(\mathbb{Q}_{p}\right)} t_{S} \chi_{S}\right)^{*} \stackrel{\text { def }}{=} \sum_{S \in \sigma\left(\mathbb{Q}_{p}\right)} \overline{t_{S}} \chi_{S},
$$

where $t_{S} \in \mathbb{C}$, having their conjugates $\overline{t_{S}}$ in $\mathbb{C}$.

Let $f \in \mathcal{M}_{p}$. Then

$$
\varphi_{p}(f)=\int_{\mathbb{Q}_{p}} f d \mu_{p}=\sum_{S \in \sigma\left(\mathbb{Q}_{p}\right)} t_{S} \mu_{p}(S),
$$

by (2.2.2).

Note that, by (2.2.4), if $S \in \sigma\left(\mathbb{Q}_{p}\right)$, then there exists a subset $\Lambda_{S}$ of $\mathbb{Z}$, such that

$$
\Lambda_{S}=\left\{j \in \mathbb{Z}: S \cap \partial_{j} \neq \varnothing\right\} .
$$

Thus, by (2.2.6) and (2.2.7), one obtains the following proposition.

Proposition 2.1. (See [2]) Let $S \in \sigma\left(\mathbb{Q}_{p}\right)$, and let $\chi_{S} \in \mathcal{M}_{p}$. Then there exist $r_{j} \in \mathbb{R}$, such that

$$
0 \leq r_{j} \leq 1 \text { in } \mathbb{R} \text {, for all } j \in \Lambda_{S},
$$

and

$$
\varphi_{p}\left(\chi_{S}\right)=\int_{\mathbb{Q}_{p}} \chi_{S} d \mu_{p}=\sum_{j \in \Lambda_{S}} r_{j}\left(\frac{1}{p^{j}}-\frac{1}{p^{j+1}}\right),
$$

where $\Lambda_{S}$ is in the sense of (2.2.7).

\section{Free Probability on $\mathcal{M}_{p}$}

Throughout this section, fix a prime $p \in \mathcal{P}$, and let $\mathbb{Q}_{p}$ be the corresponding $p$-adic number field, and let $\mathcal{M}_{p}$ be the $*$-algebra consisting of all $\mu_{p}$-measurable functions on $\mathbb{Q}_{p}$. In this section, let's establish a suitable free-probabilistic model on the *-algebra $\mathcal{M}_{p}$. Remark again that free probability provides a universal tool to study free distributions on "noncommutative" algebras, and hence, it covers the cases where given algebras are “commutative." Even though $\mathcal{M}_{p}$ is a commutative * -algebra, for our purposes, we understand our 
p-adic-analytic settings on $\mathcal{M}_{p}$ under free-probability language and terminology (See Remark 2.1).

Let $U_{k}$ be the basis elements (2.2.1) of the topology for $\mathbb{Q}_{p}$, i.e.,

$$
U_{k}=p^{k} \mathbb{Z}_{p}, \text { for all } k \in \mathbb{Z},
$$

with their boundaries

$$
\partial_{k}=U_{k} \backslash U_{k+1} \text {, for all } k \in \mathbb{Z} \text {. }
$$

Define a linear functional $\varphi_{p}: \mathcal{M}_{p} \rightarrow \mathbb{C}$ by the $p$-adic integration (2.2.2), i.e.,

$$
\varphi_{p}(f)=\int_{\mathbb{Q}_{p}} f d \mu_{p}, \text { for all } f \in \mathcal{M}_{p} .
$$

Then, by (3.2), one obtains that

$$
\varphi_{p}\left(\chi_{U_{j}}\right)=\frac{1}{p^{j}} \text {, and } \varphi_{p}\left(\chi_{\partial_{j}}\right)=\frac{1}{p^{j}}-\frac{1}{p^{j+1}},
$$

since

$$
\Lambda_{U_{j}}=\{k \in \mathbb{Z}: k \geq j\} \text {, and } \Lambda_{\partial_{j}}=\{j\},
$$

with help of (2.2.7) and (2.2.8), for all $j \in \mathbb{Z}$.

Definition 3.1. The free probability space $\left(\mathcal{M}_{p}, \varphi_{p}\right)$ is called the $p$-adic free probability space, for $p \in \mathcal{P}$, where $\varphi_{p}$ is the linear functional (3.2) on $\mathcal{M}_{p}$.

Let $U_{k}$ be in the sense of (3.1) in $\mathbb{Q}_{p}$, and $\chi_{U_{k}} \in \mathcal{M}_{p}$, for all $k \in \mathbb{Z}$. Then

$$
\chi_{U_{k_{1}}} \chi_{U_{k_{2}}}=\chi_{U_{k_{1}} \cap U_{k_{2}}}=\chi_{U_{\max \left\{k_{1}, k_{2}\right\}}},
$$

by (2.2.3), where $\max \left\{k_{1}, k_{2}\right\}$ means the maximum in $\left\{k_{1}, k_{2}\right\}$.

Say $k_{1} \leq k_{2}$ in $\mathbb{Z}$. Then $U_{k_{1}} \supseteq U_{k_{2}}$ in $\mathbb{Q}_{p}$, by (2.2.3). Therefore, $U_{k_{1}} \cap U_{k_{2}}=U_{k_{2}}$ in $\mathbb{Q}_{p}$. So, if $k_{1} \leq k_{2}$ in $\mathbb{Z}$, then

$$
\chi_{U_{k_{1}}} \chi_{U_{k_{2}}}=\chi_{U_{k_{1}} \cap U_{k_{2}}}=\chi_{U_{k_{2}}} \text { in } \mathcal{M}_{p} .
$$

Thus, one can verify that

$$
\varphi_{p}\left(\chi_{U_{k_{1}}} \chi_{U_{k_{2}}}\right)=\frac{1}{p^{\max \left\{k_{1}, k_{2}\right\}}} .
$$

Inductive to (3.3), we obtain the following result.

Proposition 3.1. (See [2]) Let $\left(j_{1}, \ldots, j_{N}\right) \in \mathbb{Z}^{N}$, for $N \in \mathbb{Z}$. Then

$$
\prod_{l=1}^{N} \chi_{U_{j_{l}}}=\chi_{U_{\max }\left\{j_{1}, \ldots, j_{N}\right\}} \text { in } \mathcal{M}_{p},
$$

and hence,

$$
\varphi_{p}\left(\prod_{l=1}^{N} \chi_{U_{j_{l}}}\right)=\frac{1}{p^{\max \left\{j_{1}, \ldots, j_{N}\right\}}} .
$$

Now, let $\partial_{k}$ be the $k$-th boundary $U_{k} \backslash U_{k+1}$ of $U_{k}$ in $\mathbb{Q}_{p}$, for all $k \in \mathbb{Z}$. Then, for $k_{1}, k_{2} \in \mathbb{Z}$, one obtains that

$$
\chi_{\partial_{k_{1}}} \chi_{\partial_{k_{2}}}=\chi_{\partial_{k_{1}} \cap \partial_{k_{2}}}=\delta_{k_{1}, k_{2}} \chi_{\partial_{k_{1}}},
$$

where $\delta$ means the Kronecker delta, and hence,

$$
\begin{aligned}
\varphi_{p}\left(\chi_{\partial_{k_{1}}} \chi_{\partial_{k_{2}}}\right) & =\delta_{k_{1}, k_{2}} \varphi_{p}\left(\chi_{\partial_{k_{1}}}\right) \\
& =\delta_{k_{1}, k_{2}}\left(\frac{1}{p^{k_{1}}}-\frac{1}{p^{k_{1}+1}}\right) .
\end{aligned}
$$

So, we obtain the following computations.

Proposition 3.2. Let $\left(j_{1}, \ldots, j_{N}\right) \in \mathbb{Z}^{N}$, for $N \in \mathbb{N}$. Then

$$
\prod_{l=1}^{N} \chi_{\partial_{j_{l}}}=\delta_{\left(j_{1}, \ldots, j_{N}\right)} \chi_{\partial_{j_{1}}} \text { in } \mathcal{M}_{p}
$$

and hence,

$$
\varphi_{p}\left(\prod_{l=1}^{N} \chi_{\partial_{j_{l}}}\right)=\delta_{\left(j_{1}, \ldots, j_{N}\right)}\left(\frac{1}{p^{j_{1}}}-\frac{1}{p^{j_{1}+1}}\right),
$$

where

$$
\delta_{\left(j_{1}, \ldots, j_{N}\right)}=\left(\prod_{l=1}^{N-1} \delta_{j_{l}, j_{l+1}}\right) \delta_{\left(j_{N}, j_{1}\right)} .
$$

Proof. The proof of (3.6) is done by (3.5).

Thus, one can get that, for any $S \in \sigma\left(\mathbb{Q}_{p}\right)$,

$$
\varphi_{p}\left(\chi_{S}\right)=\varphi_{p}\left(\sum_{j \in \Lambda_{S}} \chi_{S \cap \partial_{j}}\right)
$$

where $\Lambda_{S}$ is in the sense of (2.2.8)

$$
\begin{aligned}
& =\sum_{j \in \Lambda_{S}} \varphi_{p}\left(\chi_{S \cap \partial_{j}}\right)=\sum_{j \in \Lambda_{S}} \mu_{p}\left(S \cap \partial_{j}\right) \\
& =\sum_{j \in \Lambda_{S}} r_{j}\left(\frac{1}{p^{j}}-\frac{1}{p^{j+1}}\right),
\end{aligned}
$$

where $0 \leq r_{j} \leq 1$ are in the sense of (2.2.8), for all $j \in \mathbb{Z}$. Also, if $S_{1}, S_{2} \in \sigma\left(\mathbb{Q}_{p}\right)$, then

$$
\begin{aligned}
& \chi_{S_{1}} \chi_{S_{2}}=\left(\sum_{k \in \Lambda_{S_{1}}} \chi_{S_{1} \cap \partial_{k}}\right)\left(\sum_{j \in \Lambda_{S_{2}}} \chi_{S_{2} \cap \partial_{j}}\right) \\
& =\sum_{(k, j) \in \Lambda_{S_{1}} \times \Lambda_{S_{2}}}\left(\chi_{S_{1} \cap \partial_{k}} \chi_{S_{2} \cap \partial_{j}}\right) \\
& =\sum_{(k, j) \in \Lambda_{S_{1}} \times \Lambda_{S_{2}}} \delta_{k, j} \chi_{\left(S_{1} \cap S_{2}\right) \cap \partial_{j}} \\
& =\sum_{j \in \Lambda_{S_{1}}, \Lambda_{S_{2}}} \chi_{\left(S_{1} \cap S_{2}\right) \cap \partial_{j}},
\end{aligned}
$$

where

$$
\Lambda_{S_{1}}, \Lambda_{S_{2}}=\Lambda_{S_{1}} \cap \Lambda_{S_{2}}
$$


because $\partial_{k} \cap \partial_{j}=\delta_{k, j} \partial_{j}$.

In (3.8), it is clear that, if $\Lambda_{S_{1}, S_{2}}$ is empty, then

$\chi_{S_{1}} \chi_{S_{2}}=0_{\mathcal{M}_{p}}=\chi_{\varnothing}$, the zero element of $\mathcal{M}_{p}$,

where $\varnothing$ is the empty set in $\sigma\left(\mathbb{Q}_{p}\right)$.

Thus, one can get that there exist $w_{j} \in \mathbb{R}$, such that

$$
0 \leq w_{j} \leq 1 \text {, for all } j \in \Lambda_{S_{1}, S_{2}} \text {, }
$$

where

$$
\varphi_{p}\left(\chi_{S_{1}} \chi_{S_{2}}\right)=\sum_{j \in \Lambda_{S_{1}, S_{2}}} w_{j}\left(\frac{1}{p^{j}}-\frac{1}{p^{j+1}}\right),
$$

by (3.8) and (2.2.10), for all $S_{1}, S_{2} \in \sigma\left(\mathbb{Q}_{p}\right)$.

By (3.9), we obtain the following general result under induction.

Theorem 3.3. Let $S_{l} \in \sigma\left(\mathbb{Q}_{p}\right)$, and let $\chi_{S_{l}} \in\left(\mathcal{M}_{p}, \varphi_{p}\right)$, or $l=1, \ldots, N$. for $N \in \mathbb{N}$. Let

$$
\Lambda_{S_{1}, \ldots, S_{N}}=\bigcap_{l=1}^{N} \Lambda_{S_{l}} \text { in } \mathbb{Z},
$$

where $\Lambda_{S_{l}}$ are in the sense of (2.2.7), for $l=1, \ldots, N$. Then there exist $r_{j} \in \mathbb{R}$, such that

$$
0 \leq r_{j} \leq 1 \text { in } \mathbb{R},
$$

and

$$
\varphi_{p}\left(\prod_{l=1}^{N} \chi_{S_{l}}\right)=\sum_{j \in \Lambda_{S_{1}, . ., S_{N}}} r_{j}\left(\frac{1}{p^{j}}-\frac{1}{p^{j+1}}\right) .
$$

Proof. The proof of (3.10) is done by induction on (3.9). See [2] for details.

\section{Representations of $\left(\mathcal{M}_{p}, \varphi_{p}\right)$}

Fix a prime $p \in \mathcal{P}$. Let $\left(\mathcal{M}_{p}, \varphi_{p}\right)$ be the $p$-adic free probability space. Now, we construct a representation of $\mathcal{M}_{p}$. By understanding $\mathbb{Q}_{p}$ as a measure space, construct the $L^{2}$-space,

$$
H_{p} \stackrel{\text { def }}{=} L^{2}\left(\mathbb{Q}_{p}, \sigma\left(\mathbb{Q}_{p}\right), \mu_{p}\right)=L^{2}\left(\mathbb{Q}_{p}\right),
$$

over $\mathbb{C}$, consisting of all square-integrable $\mu_{p}$-measurable functions on $\mathbb{Q}_{p}$, Then this $L^{2}$-space is a well-defined Hilbert space equipped with its inner product $\langle,\rangle_{2}$,

$$
\left\langle f_{1}, f_{2}\right\rangle_{2} \stackrel{\text { def }}{=} \int_{\mathbb{Q}_{p}} f_{1} f_{2}^{*} d \mu_{p} \text {, for all } f_{1}, f_{2} \in H_{p} .
$$

Naturally, $H_{p}$ is the $\|\cdot\|_{2}$-norm completion in $\mathcal{M}_{p}$, where

$$
\|f\|_{2} \stackrel{\text { def }}{=} \sqrt{\langle f, f\rangle_{2}} \text {, for all } f \in H_{p},
$$

where $\langle,\rangle_{2}$ is the inner product (4.2) on $H_{p}$.

Definition 4.1. We call the Hilbert space $H_{p}$ of (4.1), the p-adic Hilbert space.

By the very construction (4.1) of the $p$-adic Hilbert space $H_{p}$, our ${ }^{*}$-algebra $\mathcal{M}_{p}$ acts on $H_{p}$, via an algebra-action $\alpha^{p}$,

$$
\alpha^{p}(f)(h)=f h, \text { for all } h \in H_{p},
$$

for all $f \in \mathcal{M}_{p}$. i.e., the morphism $\alpha^{p}$ of (4.3) is an action of $\mathcal{M}_{p}$ acting on the Hilbert space $H_{p}$. i.e., for any $f \in \mathcal{M}_{p}$, the image $\alpha^{p}(f)$ is a multiplication operator on $H_{p}$ with its symbol $f$ contained in the operator algebra $B\left(H_{p}\right)$ of all (bounded linear) operators on $H_{p}$.

Notation Denote $\alpha^{p}(f)$ by $\alpha_{f}^{p}$, for all $f \in \mathcal{M}_{p}$. Also, for convenience, denote $\alpha_{\chi_{S}}^{p}$ simply by $\alpha_{S}^{p}$, or all $S \in \sigma\left(\mathbb{Q}_{p}\right)$. For instance,

$$
\alpha_{U_{k}}^{p}=\alpha_{\chi_{U_{k}}}^{p}=\alpha^{p}\left(\chi_{U_{k}}\right)
$$

and

$$
\alpha_{\partial_{k}}^{p}=\alpha_{\chi_{\partial_{k}}}^{p}=\alpha^{p}\left(\chi_{\partial_{k}}\right)
$$

for all $k \in \mathbb{Z}$, where $U_{k}$ are in the sense of (3.1), and $\partial_{k}$ are the corresponding boundaries of $U_{k}$ in $\mathbb{Q}_{p}$, for all $k \in \mathbb{Z}$.

It is not difficult to check that

$$
\alpha_{f_{1}, f_{2}}^{p}=\alpha_{f_{1}}^{p} \alpha_{f_{2}}^{p} \text { on } H_{p} \text {, for all } f_{1}, f_{2} \in \mathcal{M}_{p},
$$

and

$$
\left(\alpha_{f}^{p}\right)^{*}=\alpha_{f} \text { on } H_{p} \text {, for all } f \in \mathcal{M}_{p} \text {. }
$$

Therefore, one obtains that:

Proposition 4.1. The pair $\left(H_{p}, \alpha^{p}\right)$ is a well-determined Hilbert-space representation of $\mathcal{M}_{p}$.

Definition 4.2. The Hilbert-space representation $\left(H_{p}, \alpha^{p}\right)$ is said to be the p-adic (Hilbert-space) representation of $\mathcal{M}_{p}$.

Depending on the $p$-adic representation of $\mathcal{M}_{p}$, one can construct the $C^{*}$-algebra $M_{p}$ in the operator algebra 
$B\left(H_{p}\right)$. Here, $B\left(H_{p}\right)$ is the operator algebra consisting of all (bounded linear) operators on $H_{p}$, equipped with the operator-norm,

$$
\|T\|=\sup \left\{\|T h\|_{2}: h \in H_{p},\|h\|_{2}=1\right\},
$$

for all $T \in B\left(H_{p}\right)$, where \|\|$_{2}$ means the $L^{2}$-norm (4.2)' on $H_{p}$.

Definition 4.3. Let $M_{p}$ be the operator-norm closure of $\mathcal{M}_{p}$ in the operator algebra $B\left(H_{p}\right)$, i.e.,

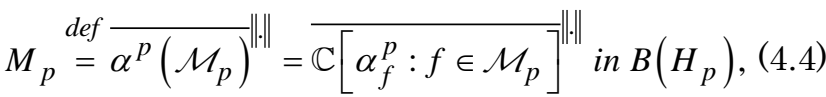

where $\bar{X}^{\|\| \|}$mean the operator-norm closures of subsets $X$ of $B\left(H_{p}\right)$. Then the $C^{*}$-algebra $M_{p}$ is called the p-adic $C^{*}$-algebra of $\left(\mathcal{M}_{p}, \varphi_{p}\right)$.

\section{Free Probability on $M_{p}$}

Throughout this section, let's fix a prime $p \in \mathcal{P}$. Let $\left(\mathcal{M}_{p}, \varphi_{p}\right)$ be the corresponding $p$-adic free probability space, and let $\left(H_{p}, \alpha^{p}\right)$ be the $p$-adic representation of $\mathcal{M}_{p}$, inducing the corresponding $p$-adic $C^{*}$-algebra $M_{p}$ of (4.4). In this section, we consider suitable free-probabilistic models on $M_{p}$. In particular, we are interested in a system $\left\{\varphi_{j}^{p}\right\}_{j \in \mathbb{Z}}$ of linear functionals on $M_{p}$, determined by the $j$-th boundaries $\left\{\partial_{j}\right\}_{j \in \mathbb{Z}}$ of $\mathbb{Q}_{p}$.

Define a linear functional $\varphi_{j}^{p}: M_{p} \rightarrow \mathbb{C}$ by a linear morphism,

$$
\varphi_{j}^{p}(a) \stackrel{\text { def }}{=}\left\langle\alpha_{a}^{p}\left(\chi_{\partial_{j}}\right), \chi_{\partial_{j}}\right\rangle_{2} \text {, for all } a \in M_{p},
$$

for all $j \in \mathbb{Z}$, where $\langle,\rangle_{2}$ is the inner product (4.2) on the $p$-adic Hilbert space $H_{p}$ of (4.1).

First, remark that, if $a \in M_{p}$, then

$$
a=\sum_{S \in \sigma\left(\mathbb{Q}_{p}\right)} t_{S} \chi_{S} \text { in } M_{p},
$$

where $\Sigma$ is finite or infinite (limit of finite) sum(s), under $C^{*}$-topology of $M_{p}$.

Definition 5.1. Let $j \in \mathbb{Z}$, and let $\varphi_{j}^{p}$ be the linear functional (5.1) on the p-adic $C^{*}$-algebra $M_{p}$. Then the $C^{*}$-probability space $\left(M_{p}, \varphi_{j}^{p}\right)$ is said to be the $j$-th (p-adic) $C^{*}$-probability space.
So, one can get the system

$$
\left\{\left(M_{p}, \varphi_{j}^{p}\right): j \in \mathbb{Z}\right\}
$$

of $C^{*}$-probability spaces for a fixed $C^{*}$-algebra $M_{p}$.

Now, fix $j \in \mathbb{Z}$, and take the corresponding $j$-th $C^{*}$-probability space $\left(M_{p}, \varphi_{j}^{p}\right)$. For $S \in \sigma\left(\mathbb{Q}_{p}\right)$, and an element $\chi_{S} \in M_{p}$, one has that

$\varphi_{j}^{p}\left(\chi_{S}\right)=\left\langle\alpha_{S}^{p}\left(\chi_{\partial_{j}}\right), \chi_{\partial_{j}}\right\rangle_{2}=\left\langle\chi_{S \cap \partial_{j}}, \chi_{\partial_{j}}\right\rangle_{2}$

$=\int_{\mathbb{Q}_{p}} \chi_{S \cap \partial_{j}} \chi_{\partial_{j}}^{*} d \mu_{p}=\int_{\mathbb{Q}_{p}} \chi_{S \cap \partial_{j}} \chi_{\partial_{j}} d \mu_{p}$

$=\int_{\mathbb{Q}_{p}} \chi_{S \cap \partial_{j}} d \mu_{p}=\mu_{p}\left(S \cap \partial_{j}\right)=r_{S}\left(\frac{1}{p^{j}}-\frac{1}{p^{j+1}}\right)$,

for some $0 \leq r_{S} \leq 1$ in $\mathbb{R}$.

Proposition 5.1. Let $S \in \sigma\left(\mathbb{Q}_{p}\right)$, and $\alpha_{S}^{p}=\alpha_{\chi S}^{p}$ $\in\left(M_{p}, \varphi_{j}^{p}\right)$, for a fixed $j \in \mathbb{Z}$. Then there exists $r_{S} \in \mathbb{R}$, such that

$$
0 \leq r_{S} \leq 1 \text { in } \mathbb{R}
$$

and

$$
\varphi_{j}\left(\left(\alpha_{S}^{p}\right)^{n}\right)=r_{S}\left(\frac{1}{p^{j}}-\frac{1}{p^{j+1}}\right), \text { for all } n \in \mathbb{N} .
$$

Proof. Remark that the element $\alpha_{S}$ is a projection in $M_{p}$, in the sense that:

$$
\left(\alpha_{S}^{p}\right)^{*}=\alpha_{S}^{p}=\left(\alpha_{S}^{p}\right)^{2} \text {, in } M_{p}
$$

So,

$$
\left(\alpha_{S}^{p}\right)^{n}=\alpha_{S}^{p} \text {, for all } n \in \mathbb{N} \text {. }
$$

Thus, by (5.2), we obtain (5.3).

The above proposition characterizes the free distributions of $\alpha_{S}$ in the $j$-th $C^{*}$-probability space $\left(M_{p}, \varphi_{j}^{p}\right)$, for $j \in \mathbb{Z}$.

More precisely, we obtain the following theorem.

Theorem 5.2. Let $S_{l} \in \sigma\left(\mathbb{Q}_{p}\right)$, and $\alpha_{S_{l}}^{p}=\alpha^{p}\left(\chi_{S_{l}}\right)$ $\in\left(M_{p}, \varphi_{j}^{p}\right)$, for a fixed $j \in \mathbb{Z}, \quad$ for $l=1, \ldots, N$, for $N \in \mathbb{N}$. Then there exists $r_{\left(S_{1}, \ldots, S_{N}\right)} \in \mathbb{R}$, such that

$$
0 \leq r_{\left(S_{1}, \ldots, S_{N}\right)} \leq 1 \text { in } \mathbb{R} \text {, }
$$

and

$$
\varphi_{j}\left(\left(\prod_{l=1}^{N} \alpha_{S_{l}}^{p}\right)^{n}\right)=r_{\left(S_{1}, \ldots, S_{N}\right)}\left(\frac{1}{p^{j}}-\frac{1}{p^{j+1}}\right),
$$

for all $n \in \mathbb{N}$. 
Proof. Let $S_{1}, \ldots, S_{N}$ be $\mu_{p}$-measurable subsets of $\mathbb{Q}_{p}$, for $N \in \mathbb{N}$, and let

$$
S=\bigcap_{l=1}^{N} S_{l} \in \sigma\left(\mathbb{Q}_{p}\right) .
$$

Then, one has that

$$
\alpha_{S}^{p}=\prod_{l=1}^{N} \alpha_{S_{l}}^{p} \text { in } M_{p},
$$

satisfying

$$
\left(\alpha_{S}^{p}\right)^{*}=\alpha_{S}=\left(\alpha_{S}^{p}\right)^{2} \text { in } M_{p}
$$

Therefore, by (5.3), the formula (5.4) holds.

The above joint free-moment formula (5.4) characterizes the free-distributions of finitely many projections $\alpha_{S_{1}}, \ldots, \alpha_{S_{N}}$ in the $j$-th $C^{*}$-probability space $\left(M_{p}, \varphi_{j}^{p}\right)$, for $j \in \mathbb{Z}$.

As corollaries of (5.4), we obtain the following results. Corollary 5.3. Let $U_{k}$ be in the sense of (3.1), and $\partial_{k}$, the $k$-th boundaries of $U_{k}$ in $\mathbb{Q}_{p}$, for all $k \in \mathbb{Z}$. Then

$$
\varphi_{j}^{p}\left(\left(\alpha_{U_{k}}^{p}\right)^{n}\right)= \begin{cases}\frac{1}{p^{j}}-\frac{1}{p^{j+1}} & \text { if } k \leq j \\ 0 & \text { otherwise, }\end{cases}
$$

and

$$
\varphi_{j}^{p}\left(\left(\alpha_{\partial_{k}}^{p}\right)^{n}\right)=\delta_{j, k}\left(\frac{1}{p^{j}}-\frac{1}{p^{j+1}}\right)
$$

for all $n \in \mathbb{N}$, for $k \in \mathbb{Z}$.

\section{Semigroup $C^{*}$-Subalgebras $\mathfrak{S}_{p}$ of $\mathcal{M}_{p}$}

Let $M_{p}$ be the $p$-adic $C^{*}$-algebra for $p \in \mathcal{P}$, as in Section 5. Take operators

$$
P_{p, j}=\alpha_{\partial_{j}}^{p} \in M_{p},
$$

for all $j \in \mathbb{Z}$, for $p \in \mathcal{P}$.

As we have seen, these operators $P_{p, j}$ are projections on the $p$-adic Hilbert space $H_{p}$ in $M_{p}$, i.e.,

$$
P_{p, j}^{*}=P_{p, j}=P_{p, j}^{2}
$$

for all $p \in \mathcal{P}, \quad j \in \mathbb{Z}$. We now restrict our interests to these projections $P_{p, j}$ of (6.1).

Definition 6.1. Fix $p \in \mathcal{P}$. Let $\mathfrak{S}_{p}$ be the $C^{*}$-subalgebra

$$
\mathcal{S}_{p}=C^{*}\left(\left\{P_{p, j}\right\}_{j \in \mathbb{Z}}\right)=\overline{\mathbb{C}\left[\left\{P_{p, j}\right\}_{j \in \mathbb{Z}}\right]} \text { of } M_{p}
$$

where $P_{p, j}$ are projections (6.1), for all $j \in \mathbb{Z}$. We call this $C^{*}$-subalgebra $\mathfrak{S}_{p}$, the p-adic boundary ( $C^{*}$-)subalgebra of $M_{p}$.

We originally defined such $p$-adic boundary subalgebras $\mathcal{S}_{p}$ in [4].

Proposition 6.1. (See [4]) Let $\mathfrak{S}_{p}$ be the p-adic boundary subalgebra (6.2) of the p-adic $C^{*}$-algebra $M_{p}$. Then

$$
\mathcal{S}_{p} \stackrel{*-i s o}{=} \underset{j \in \mathbb{Z}}{\oplus}\left(\mathbb{C} \cdot P_{p, j}\right)^{*-i s o}=\mathbb{C}^{\oplus \mathbb{Z}},
$$

in $M_{p}$.

By the structure theorem (6.3) of $\mathfrak{S}_{p}$, it acts like a diagonal subalgebra inside $M_{p}$. Since p-adic boundary subalgebras $\mathfrak{S}_{p}$ are $C^{*}$-subalgebras of $M_{p}$, one can naturally get the $C^{*}$-probability spaces

$$
\left(\mathfrak{S}_{p}, \varphi_{j}^{p}\right) \text {, for all } p \in \mathcal{P} \text { and } j \in \mathbb{Z} \text {. }
$$

i.e., we have a family

$$
\left\{\left(\mathcal{S}_{p}, \varphi_{j}^{p}\right): p \in \mathcal{P}, j \in \mathbb{Z}\right\}
$$

where the linear functional $\varphi_{j}^{p}$ are restrict linear functional $\left.\varphi_{j}^{p}\right|_{\mathfrak{S}_{p}}$ of $\varphi_{j}^{p}$ on $M_{p}$, for all $j \in \mathbb{Z}$, and $p \in \mathcal{P}$.

\section{Weighted-Semicircularity}

Let $M_{p}$ be the $p$-adic $C^{*}$-algebra, and let $\mathcal{S}_{p}$ be the boundary subalgebra (8.2) of $M_{p}$, satisfying the structure theorem (6.3):

$$
\mathcal{S}_{p}=C^{*}\left(\left\{P_{p, j}\right\}_{j \in \mathbb{Z}}\right)^{*-i s o}=\mathbb{C}^{\oplus|\mathbb{Z}|},
$$

where $P_{p, j}$ are projections $\alpha_{\partial_{j}}^{p}$ of (6.1) on $H_{p}$, for all $j \in \mathbb{Z}$.

Then we have

$$
\begin{aligned}
& \varphi_{j}^{p}\left(P_{p, k}\right)=\delta_{j, k}\left(\frac{1}{p^{j}}-\frac{1}{p^{j+1}}\right), \forall p \in \mathcal{P}, \\
& \text { and } j, k \in \mathbb{Z} \text {. }
\end{aligned}
$$

Now, let $\phi$ be the Euler totient function, which is an arithmetic function

$$
\phi: \mathbb{N} \rightarrow \mathbb{C}
$$

defined by

$$
\phi(n)=|\{k \in \mathbb{N}: k \leq n, \operatorname{gcd}(n, k)=1\}|,
$$


for all $n \in \mathbb{N}$, where $|X|$ mean the cardinalities of sets $X$, and gcd means the greatest common divisor.

It is well-known that

$$
\phi(n)=n\left(\prod_{q \in \mathcal{P}, q \mid n}\left(1-\frac{1}{q}\right)\right)
$$

for all $n \in \mathbb{N}$, where $q \mid n$ means " $q$ divides $n$," or " $q$ is a divisor of $n$."

Thus, one has

$$
\phi(p)=p-1=p\left(1-\frac{1}{p}\right), \text { for all } p \in \mathcal{P},
$$

by (7.0.2).

So, one can get that

$\varphi_{j}^{p}\left(P_{p, j}\right)=\frac{1}{p^{j}}\left(1-\frac{1}{p}\right)=\frac{p}{p^{j+1}}\left(1-\frac{1}{p}\right)=\frac{\phi(p)}{p^{j+1}},(7.0 .4)$

by (7.0.1) and (7.0.3), for $P_{p, j} \in \mathcal{S}_{p}$, for all $p \in \mathcal{P}$, $j \in \mathbb{Z}$.

Now, Define new linear functionals

$$
\tau_{j}^{p}: \mathfrak{S}_{p} \rightarrow \mathbb{C}
$$

by linear morphisms satisfying that

$$
\tau_{j}^{p}\left(P_{p, k}\right)=\frac{\delta_{j . k}}{\phi(p)} \varphi_{j}^{p}\left(P_{p, j}\right)=\frac{\delta_{j . k}}{p^{j+1}},
$$

for all $p \in \mathcal{P}$, and $j, k \in \mathbb{Z}$, inducing new $C^{*}$-probability spaces

$$
\mathfrak{S}_{p}(j)=\left(\mathfrak{S}_{p}, \tau_{j}^{p}\right) \text {, for } p \in \mathcal{P}, j \in \mathbb{Z}
$$

as in (6.4), where $\tau_{j}^{p}$ are in the sense of (7.0.5).

Proposition 7.1. Let $\mathfrak{S}_{p}(j)=\left(\mathfrak{S}_{p}, \tau_{j}^{p}\right)$ be $a$ $C^{*}$-probability space (7.0.6), and let $P_{p, k}$ be generating projections of $\mathfrak{S}_{p}$, for all $j \in \mathbb{Z}$. Then

$$
\tau_{j}^{p}\left(P_{p, k}^{n}\right)=\frac{\delta_{j, k}}{p^{j+1}}, \text { for all } n \in \mathbb{N} .
$$

Proof. The free-moment formula (7.0.7) is proven by (7.0.5), because $P_{p, k}$ are projections in $\mathfrak{S}_{p}$.

\subsection{Semicircular and Weighted-Semicircular Elements}

Let $(A, \varphi)$ be an arbitrary topological *-probability space ( $C^{*}$-probability space, or $W^{*}$-probability space, or Banach *-probability space, etc.) equipped with a topological *-algebra A (C_-algebra, resp., W_-algebra, resp., Banach *-algebra), and a bounded linear functional $\varphi$ on $A$.
Definition 7.1. Let a be a self-adjoint operator in $(A, \varphi)$. It is said to be even in $(A, \varphi)$, if all odd free moments of a vanish, i.e.,

$$
\varphi\left(a^{2 n-1}\right)=0 \text {, for all } n \in \mathbb{N} .
$$

Let a be a "self-adjoint" operator of $(A, \varphi)$ satisfying the even-ness (7.1.1). Then it is said to be semicircular in $(A, \varphi)$, if

$$
\varphi\left(a^{2 n}\right)=c_{n}, \text { for all } n \in \mathbb{N}
$$

where $c_{n}$ are the $n$-th Catalan number,

$$
c_{n}=\frac{1}{n+1}\left(\begin{array}{c}
2 n \\
n
\end{array}\right)=\frac{1}{n+1} \frac{(2 n) !}{(n !)^{2}}=\frac{(2 n) !}{n !(n+1) !},
$$

for all $n \in \mathbb{N}$.

It is well-known that, if $k_{n}(\ldots)$ is the free cumulant on $A$ in terms of $\varphi$ (in the sense of [12]), then a self-adjoint operator a is semicircular in $(A, \varphi)$, if and only if

$$
k_{n}(\underbrace{a, a, \ldots \ldots, a}_{n \text {-times }})=\left\{\begin{array}{l}
1 \text { if } n=2 \\
0 \text { otherwise, }
\end{array}\right.
$$

for all $n \in \mathbb{N}$ (e.g., see [11,14], and cited papers therein). The above characterization (7.1.3) is obtained by the Möbius inversion of [12].

Thus, the semicircular operators a of $(A, \varphi)$ can be re-defined by the self-adjoint operators satisfying the free-cumulant characterization (7.1.3).

Motivated by (7.1.3), one can define so-called the weighted-semicircular elements.

Definition 7.2. Let $a \in(A, \varphi)$ be a self-adjoint operator. It is said to be weighted-semicircular in $(A, \varphi)$ with its weight $t_{0}$ (in short, $t_{0}$-semicircular), if there exists $t_{0} \in \mathbb{C}$, such that

$$
k_{n}(\underbrace{a, a, \ldots \ldots, a}_{n \text {-times }})= \begin{cases}t_{0} & \text { if } n=2 \\ 0 & \text { otherwise }\end{cases}
$$

for all $n \in \mathbb{N}$, where $k_{n}(\ldots)$ is the free cumulant in terms of $\varphi$ in the sense of [12].

By the definition (7.1.4), and by the Möbius inversion, we obtained the following free-moment characterization of (7.1.4) in [4]: A self-adjoint operator $a$ in a *-probability space $(A, \varphi)$ is to-semicircular, if and only if (i) it is even in $(A, \varphi)$, and (ii) there exists $t_{0} \in \mathbb{C}$, such that

$$
\varphi\left(a^{n}\right)= \begin{cases}t_{0}^{\frac{n}{2}} c_{\frac{n}{2}} & \text { if } n \text { is even } \\ 0 & \text { if } n \text { is odd, }\end{cases}
$$


for all $n \in \mathbb{N}$, where $c_{m}$ mean the $m$-th Catalan numbers, for all $m \in \mathbb{N}$.

\subsection{Tensor Product Banach *-Algebra $\mathfrak{L S}_{p}$}

Let $\mathfrak{S}_{p}(k)=\left(\mathfrak{S}_{p}, \tau_{k}^{p}\right)$ be a $C^{*}$-probability space (7.0.6), for $p \in \mathcal{P}, k \in \mathbb{Z}$. Throughout this section, we fix $k$ in $\mathbb{Z}$, and the corresponding $C^{*}$-probability space $\mathfrak{S}_{p}(k)$.

Define now a Banach-space operators (or bounded linear transformations) $c_{p}$ and $a_{p}$ "acting on the $C^{*}$-algebra $\mathfrak{S}_{p}$, ” by linear morphisms satisfying,

$$
c_{p}\left(P_{p, j}\right)=P_{p, j+1} \text {, and } a_{p}\left(P_{p, j}\right)=P_{p, j-1},
$$

on $\mathfrak{S}_{p}$, for all $j \in \mathbb{Z}$.

Definition 7.3. The Banach-space operators $c_{p}$ and $a_{p}$ on $\mathfrak{S}_{p}$ in the sense of (7.2.1) are called the p-creation, respectively, the $p$-annihilation on $\mathfrak{S}_{p}$, for $p \in \mathcal{P}$. Define a new Banach-space operator $l_{p}$ by

$$
l_{p}=c_{p}+a_{p} \text { on } \mathcal{S}_{p} .
$$

We call it the p-radial operator on $\mathfrak{S}_{p}$.

Let $l_{p}$ be the $p$-radial operator $c_{p}+a_{p}$ of (7.2.2) acting on $\mathfrak{S}_{p}$. Construct a Banach algebra $\mathfrak{L}_{p}$ by

$$
\mathfrak{L}_{p}=\overline{\mathbb{C}\left[l_{p}\right]} \text { in } B\left(\mathfrak{S}_{p}\right),
$$

where $B\left(\mathfrak{S}_{p}\right)$ means the operator space consisting of all bounded linear transformations on $\mathfrak{S}_{p}$, equipped with its operator-norm \|.\|, defined by

$$
\|T\|=\sup \left\{\|T x\|_{\mathfrak{S}_{p}}: x \in \mathfrak{S}_{p} \text { s.t., }\|x\|_{\mathfrak{S}_{p}}=1\right\} \text {, }
$$

where

$$
\|x\|_{\mathfrak{S}_{p}}=\sup \left\{\|x(h)\|_{2}: h \in H_{p} \text { s.t., }\|h\|_{2}=1\right\},
$$

giving the $C^{*}$-norm topology for $\mathfrak{S}_{p}$ (which is the subspace topology of that for $M_{p}$ ), where \|\|$_{2}$ means the Hilbert-space $L^{2}$-norm on the $p$-adic Hilbert space $H_{p}=L^{2}\left(\mathbb{Q}_{p}\right)$, for all $p \in \mathcal{P}$.

On $\mathfrak{L}_{p}$, define the adjoint $\left({ }^{*}\right)$ by

$$
\sum_{k=0}^{\infty} s_{k} l_{p}^{k} \in \mathfrak{L}_{p} \mapsto \sum_{k=0}^{\infty} \overline{s_{k}} l_{p}^{k} \in \mathfrak{L}_{p},
$$

where $s_{k} \in \mathbb{C}$, with their conjugates $\overline{s_{k}} \in \mathbb{C}$. It is indeed a well-defined adjoint on $\mathfrak{L}_{p}$ (See [4]).
Then, equipped with the adjoint (7.2.4), this Banach algebra $\mathfrak{L}_{p}$ of (7.2.3) forms a Banach *-algebra.

Definition 7.4. Let $\mathfrak{L}_{p}$ be a Banach *-algebra (7.2.3) in $B\left(\mathfrak{S}_{p}\right)$ for $p \in \mathcal{P}$ We call $\mathfrak{L}_{p}$, the p-radial (Banach-*-) algebra on $\mathfrak{S}_{p}$.

Let $\mathfrak{L}_{p}$ be the p-radial algebra on $\mathfrak{S}_{p}$. Construct now the tensor product *-algebra $\mathfrak{L S}_{p}$ by

$$
\mathfrak{L} \mathfrak{S}_{p}=\mathfrak{L}_{p} \otimes_{\mathbb{C}} \mathfrak{S}_{p},
$$

where $\otimes_{\mathbb{C}}$ means the tensor product of Banach *-algebras.

Take now a generating element $l_{p}^{n} \otimes P_{p, j}$, for some $n \in \mathbb{N}_{0}, \quad j \in \mathbb{Z}$, where $P_{p, j}$ are in the sense of (6.1) in $\mathfrak{S}_{p}$, and

$$
\mathbb{N}_{0}=\mathbb{N} \bigcup\{0\},
$$

with axiomatization: $l_{p}^{0}=1_{\mathfrak{L}_{p}}$, the identity operator of $\mathfrak{L}_{p}$ on $\mathfrak{S}_{p}$, satisfying

$$
1_{\mathfrak{L}_{p}}\left(P_{p, j}\right)=P_{p, j}, \text { for all } P_{p, j} \in \mathfrak{S}_{p},
$$

for all $j \in \mathbb{Z}$.

Define now a bounded linear morphism

$$
E_{p}: \mathfrak{L} \mathfrak{S}_{p} \rightarrow \mathfrak{S}_{p}
$$

by a linear transformation satisfying that:

$$
E_{p}\left(l_{p}^{k} \otimes P_{p, j}\right)=\frac{\left(p^{j+1}\right)^{k+1}}{\left[\frac{k}{2}\right]+1} l_{p}^{k}\left(P_{p, j}\right),
$$

for all $k \in \mathbb{N}_{0}, \quad j \in \mathbb{Z}$, where $\left[\frac{k}{2}\right]$ is the minimal integer greater than or equal to $\frac{k}{2}$, for all $k \in \mathbb{N}_{0}$.

By the cyclicity of the tensor factor $\mathfrak{L}_{p}$ of $\mathfrak{L S}_{p}$, and by the fact that: all generating elements $\left\{P_{p, j}\right\}_{j \in \mathbb{Z}}$ of $\mathfrak{S}_{p}$ are mutually orthogonal projections, the above morphism $E_{p}$ is well-defined as a linear transformation.

Now, consider how our $p$-radial operator $l_{p}=c_{p}+a_{p}$ acts on $\mathfrak{S}_{p}$. First observe that if $c_{p}$ and $a_{p}$ are the $p$-creation, respectively, the $p$-annihilation on $\mathfrak{S}_{p}$, then

$$
c_{p} a_{p}\left(P_{p, j}\right)=P_{p, j}=a_{p} c_{p}\left(P_{p, j}\right),
$$

for all $j \in \mathbb{Z}, p \in \mathcal{P}$.

Lemma 7.2. Let $c_{p}, a_{p}$ be the p-creation, respectively, the p-annihilation on $\mathfrak{S}_{p}$. Then

$$
c_{p} a_{p}=1_{\mathfrak{S}_{p}}=a_{p} c_{p},
$$


where $1_{\mathfrak{S}_{p}}$ is the identity operator on $\mathfrak{S}_{p}$.

Proof. The formula (7.2.8) holds by (6.3) and (7.2.7).

Also, the formula (7.2.8) shows that the Banach-space operators $c_{p}$ and $a_{p}$ are acting commutatively on $\mathfrak{S}_{p}$. Therefore, one can obtain that

$$
l_{p}^{n}=\left(c_{p}+a_{p}\right)^{n}=\sum_{k=0}^{n}\left(\begin{array}{l}
n \\
k
\end{array}\right) c_{p}^{k} a_{p}^{n-k},
$$

with identity:

$$
c_{p}^{0}=1_{\mathfrak{S}_{p}}=a_{p}^{0}
$$

for all $n \in \mathbb{N}$, where

$$
\left(\begin{array}{l}
n \\
k
\end{array}\right)=\frac{n !}{k !(n-k) !} \text {, for all } n \in \mathbb{N}, k \in \mathbb{N}_{0} .
$$

By considering the formulas (7.2.8) and (7.2.9) together, one obtains the following proposition.

Proposition 7.3. Let $l_{p} \in \mathcal{L}_{p}$ be the p-radial operator on $\mathfrak{S}_{p}$. Then

$$
\begin{aligned}
& l_{p}^{2 m-1} \text { does not contain } 1_{\mathfrak{S}_{p}}-\text { term, and } \\
& l_{p}^{2 m} \text { contains its } 1_{\mathfrak{S}_{p}}-\text { term, }\left(\begin{array}{c}
2 m \\
m
\end{array}\right) \cdot 1_{\mathfrak{S}_{p}},
\end{aligned}
$$

for all $m \in \mathbb{N}$.

Proof. The proofs of (7.2.10) and (7.2.11) are done by straightforward computations under (7.2.8) and (7.2.9). See [4] for more details.

\subsection{Weighted-Semicircular Elements $Q_{p, j}$ in $\mathfrak{L} \mathfrak{S}_{p}$}

Fix $p \in \mathcal{P}$, and let $\mathfrak{L S}_{p}$ be the tensor product Banach *-algebra $\mathfrak{L}_{p} \otimes_{\mathbb{C}} \mathcal{S}_{p}$ in the sense of (7.2.5), and let $E_{p}: \mathfrak{L} \mathfrak{S}_{p} \rightarrow \mathfrak{S}_{p}$ be the linear transformation of (7.2.6). Throughout this section, fix an element

$$
Q_{p, j}=l_{p} \otimes P_{p, j} \in \mathfrak{L} \mathfrak{S}_{p}, \text { for } j \in \mathbb{Z},
$$

where $P_{p, j}$ are projections (6.1), generating $\mathfrak{S}_{p}$. Observe that

$$
Q_{p, j}^{n}=\left(l_{p} \otimes P_{p, j}\right)^{n}=l_{p}^{n} \otimes P_{p, j}^{n}=l_{p}^{n} \otimes P_{p, j},(7.3
$$

for all $n \in \mathbb{N}$, for all $j \in \mathbb{Z}$. By (7.3.2), one can realize that the operators $Q_{p, j}$ of (7.3.1) generate $\mathfrak{L S}_{p}$, for all $j \in \mathbb{Z}$.

Consider that, if $Q_{p, j} \in \mathfrak{L} \mathfrak{S}_{p}$ is in the sense of (7.3.1), for $j \in \mathbb{Z}$, then

$$
E_{p}\left(Q_{p, j}^{n}\right)=E_{p}\left(l_{p}^{n} \otimes P_{p, j}\right)=\frac{\left(p^{j+1}\right)^{n+1}}{\left[\frac{n}{2}\right]+1} l_{p}^{n}\left(P_{p, j}\right)
$$

by (7.2.6) and (7.3.2), for all $n \in \mathbb{N}$.

Now, for any fixed $j \in \mathbb{Z}$, define a linear functional $\tau_{p, j}^{0}$ on $\mathfrak{L S}_{p}$ by

$$
\tau_{p, j}^{0}=\tau_{j}^{p} \circ E_{p} \text { on } \mathfrak{L S}_{p},
$$

where $\tau_{j}^{p}$ is in the sense of (7.0.5).

By the linearity of both $\tau_{j}^{p}$ and $E_{p}$, the morphism $\tau_{p, j}^{0}$ of (7.3.4) is a well-defined linear functional on $\mathfrak{L S}_{p}$. So, the pair $\left(\mathfrak{L S}_{p}, \tau_{p, j}^{0}\right)$ forms a Banach *probability space in the sense of [12] and [14].

By (7.3.3) and (7.3.4), if $Q_{p, j}$ is in the sense of (7.3.1), then

$$
\tau_{p, j}^{0}\left(Q_{p, j}^{n}\right)=\frac{\left(p^{j+1}\right)^{n+1}}{\left[\frac{n}{2}\right]+1} \tau_{j}^{p}\left(l_{p}^{n}\left(P_{p, j}\right)\right),
$$

for all $n \in \mathbb{N}$.

Theorem 7.4. Let $Q_{p, j}=l_{p} \otimes P_{p, j} \in\left(\mathfrak{L S}_{p}, \tau_{p, j}^{0}\right)$, for $a$ fixed $j \in \mathbb{Z}$. Then $Q_{p, j}$ is $p^{2(j+1)}$-semicircular in $\left(\mathfrak{L} \mathfrak{S}_{p}, \tau_{p, j}^{0}\right)$. More precisely, one obtains that

$$
\tau_{p, j}^{0}\left(Q_{p, j}^{n}\right)= \begin{cases}c_{\frac{n}{2}}\left(p^{2(j+1)}\right)^{\frac{n}{2}} & \text { if } n \text { is even } \\ 0 & \text { if } n \text { is odd },\end{cases}
$$

for all $n \in \mathbb{N}$. Equivalently, if $k_{n}^{0, p, j}(\ldots)$ means a free cumulant in terms of the linear functional $\tau_{p, j}^{0}$ of (9.3.5) on $\mathfrak{L S}_{p}$, then

$k_{n}^{0, p, j}(\underbrace{Q_{p, j}, Q_{p, j}, \ldots, Q_{p, j}}_{n \text {-times }})= \begin{cases}\left(p^{j+1}\right)^{2} & \text { if } n=2 \\ 0 & \text { otherwise, }\end{cases}$

for all $n \in \mathbb{N}$.

Proof. The formula (7.3.6) is proven by the straightforward computations from (7.3.5), with help of (7.2.10) and (7.2.11). Also, the formula (7.4.7) is obtained by the Möbius inversion of [12] from (7.3.6). See [4] for more details.

\section{Semicircularity on $\mathfrak{L} \mathfrak{S}$}

For all $p \in \mathcal{P}, j \in \mathbb{Z}$, let $\mathfrak{L S}_{p}=\mathfrak{L}_{p} \otimes_{\mathbb{C}} \mathfrak{S}_{p}$, and $\tau_{p, j}^{0}$ be in the sense of (7.2.5), respectively, (7.3.4). Then, one has the corresponding Banach *-probability spaces,

$$
\mathfrak{L} \mathfrak{S}_{p}(j)=\left(\mathfrak{L} \mathfrak{S}_{p}, \tau_{p, j}^{0}\right)
$$


for all $p \in \mathcal{P}, j \in \mathbb{Z}$.

Let $Q_{p, k}=l_{p} \otimes P_{p, k}$ be the generating elements (7.3.1) of $\mathfrak{L S}_{p}(j)$, for $p \in \mathcal{P}, k \in \mathbb{Z}$, where $\mathfrak{L S}_{p}(j)$ are in the sense of (8.0.1). Then a generating element $Q_{p, j}$ of $\mathfrak{L} \mathfrak{S}_{p}(j)$ is $p^{2(j+1)}$-semicircular in $\mathfrak{L S}_{p}(j)$, by (7.3.6) and (7.3.7). i.e.,

$$
k_{n}^{0, p, j}\left(Q_{p, j}, Q_{p, j}, \ldots, Q_{p, j}\right)= \begin{cases}p^{2(j+1)} & \text { if } n=2 \\ 0 & \text { otherwise, }\end{cases}
$$

and

$$
\tau_{p, j}^{0}\left(Q_{p, j}^{n}\right)=\omega_{n}\left(p^{2(j+1)}\right)^{\frac{n}{2}} c_{\frac{n}{2}},
$$

for all $p \in \mathcal{P}, j \in \mathbb{Z}$, for all $n \in \mathbb{N}$, where

$$
\omega_{n}=\left\{\begin{array}{l}
1 \text { if } n \text { is even } \\
0 \text { if } n \text { is odd, }
\end{array}\right.
$$

for all $n \in \mathbb{N}$.

\subsection{Free Product *-Probability Spaces}

Let $\left(A_{k}, \varphi_{k}\right)$ be arbitrary topological *-probability spaces (e.g., $C^{*}$-probability spaces, or $W^{*}$-probability spaces, or Banach *-probability spaces, etc.) consisting of topological *-algebras $A_{k}$ (e.g., $C^{*}$-algebras, or $W^{*}$-algebras, or Banach *-algebras, etc.), and corresponding bounded linear functional $\varphi_{k}$, for $k \in \Delta$, where $\Delta$ is an arbitrary countable (finite or infinite) index set.

The free product topological *-probability space $(A, \varphi)$ of $\left\{\left(A_{k}, \varphi_{k}\right)\right\}_{k \in \Delta}$ is a new topological *-probability space, consisting of the free product topological *-algebra

$$
A=\underset{l \in \Delta}{\star} A_{l}
$$

generated by the noncommutative reduced words, called free reduced words, in $\bigcup_{l=1}^{N} A_{l}$ (under product topology), having a new linear functional

$$
\varphi=\underset{l \in \Delta}{\star} \varphi_{l},
$$

where $\varphi$ satisfies that: if $a_{i_{1}} a_{i_{2}} \ldots a_{i_{n}}$ is a free reduced word in $A$, then

$$
\varphi\left(a_{i_{1}} a_{i_{2}} \ldots a_{i_{n}}\right)=\varphi_{i_{1}}\left(a_{i_{1}}\right) \varphi_{i_{2}}\left(a_{i_{2}}\right) \ldots \varphi_{i_{n}}\left(a_{i_{n}}\right),
$$

for all $\left(i_{1}, \ldots, i_{n}\right) \in \Delta^{n}$, for all $n \in \mathbb{N}$ (e.g., see $[11,12,14]$ ).

We denote the above relations together by

$$
(A, \varphi)=\underset{k \in \Delta}{\star}\left(A_{k}, \varphi_{k}\right)=\left(\underset{k \in \Delta}{\star} A_{k}, \underset{k \in \Delta}{\star} \varphi_{k}\right) .
$$

The topological *-algebra $A=\underset{k \in \Delta}{\star} A_{k}$ is understood as a Banach space,

$$
\left.\mathbb{C} \oplus\left(\underset{n=1}{\oplus}\left(\begin{array}{c}
\oplus \\
\left(i_{1}, \ldots, i_{n}\right) \in a l t \\
\left.\oplus \Delta^{n}\right)
\end{array} \underset{k=1}{\oplus} A_{i_{k}}^{o}\right)\right)\right)
$$

with

$$
A_{i_{k}}^{o}=A_{i_{k}} \ominus \mathbb{C} \text {, for all } k=1, \ldots, n,
$$

where

$$
\operatorname{alt}\left(\Delta^{n}\right)=\left\{\begin{array}{l|l}
\left(i_{1}, \ldots, i_{n}\right) & \begin{array}{l}
\left(i_{1}, \ldots, i_{n}\right) \in^{n} \\
i_{1} \neq i_{2}, i_{2} \neq i_{3} \\
\ldots, i_{n-1} \neq i_{n}
\end{array}
\end{array}\right\},
$$

for all $n \in \mathbb{N}$, and where $\oplus$ and $\otimes$ are the direct product, respectively, the tensor product of Banach spaces (e.g., see $[12,14])$.

In particular, if an element $a \in A$ is of the form of free reduced word,

$$
\prod_{l=1}^{n} a_{i_{l}} \text { in } A
$$

with

$$
\left(i_{1}, \ldots, i_{n}\right) \in \operatorname{alt}\left(\Delta^{n}\right)
$$

then one can understand $a$ as an equivalent Banach-space vector

$$
\bigotimes_{l=1}^{n} a_{i_{l}} \text { in the Banach space } A \text { of (8.1.1), }
$$

contained in the minimal direct summand $\underset{k=1}{\otimes} A_{i_{k}}^{o}$ (which is a closed subspace) of $A$ in (8.1.1).

We denote this relation by

$$
a \stackrel{\text { equi }}{=} \underset{l=1}{\otimes} a_{i_{l}} \in \underset{k=1}{\otimes} A_{i_{k}}^{o} \text { in } A .
$$

Of course, the left-hand side $a$ of (8.1.2) means the operator in $A$, while, the right-hand side $\otimes_{l=1}^{n} a_{i_{l}}$ means the Banach-space vector in the direct summand $\underset{k=1}{\otimes} A_{i_{k}}^{o}$ of the Banach space $A$ of (8.1.1). Note that, under same argument, one may understand $a$ as an operator $\otimes_{l=1}^{n} a_{i_{l}}$ in the *-subalgebra

$$
\otimes_{k=1}^{n} A_{i_{k}} \text { of } A
$$

where $\otimes_{\mathbb{C}}$ means the tensor product of topological $*$-algebras. Notation 8.1. In the rest of this paper, we will call the above *-subalgebra $\underset{k=1}{\otimes_{\mathbb{C}}} A_{i_{k}}$ of $A$, the minimal free summand of A containing a given free reduced word $a=\prod_{l=1}^{n} a_{i_{l}}$. 
Remark that, if $a$ is a free reduced word in $A$, then

$$
a^{k} \stackrel{\text { equi }}{=}\left(\begin{array}{c}
n \\
\otimes \\
l=1
\end{array} a_{i_{l}}\right)^{k}=\bigotimes_{l=1}^{n} a_{i_{l}^{k}}^{k}=\prod_{l=1}^{\text {equi }} a_{i_{l}}^{n}
$$

in the minimal free summand of $A$ containing $a$ in the sense of Notation 8.1, for all $k \in \mathbb{N}$. Remark also that even though the above relation (8.1.3) holds inside the minimal free summand, it does not hold fully in $A$, in general (in particular, whenever $k>1$ ).

Notation and Remark 8.1. (From below, NR 8.1) Let $a=\prod_{l=1}^{n} a_{i_{l}}$ be a free reduced word in $A$, as above. The power $a^{k}$ in (8.1.3) means the $k$-th power of $a$ as an element of the minimal free summand of $A$ containing $a$.

To avoid the confusion, we will use the notation $a^{(k)}$, as a construction of new free "non-reduced" word,

$$
a^{(k)}=\underbrace{a \ldots a \ldots a}_{k-\text { times }}
$$

in $A$.

For example, let $a=a_{i_{1}} a_{i_{2}} a_{i_{1}}$ be a free reduced word with

$$
\left(i_{1}, i_{2}, i_{1}\right) \in \operatorname{alt}\left(\Delta^{3}\right)
$$

which is equivalent to

$$
a_{i_{1}} \otimes a_{i_{2}} \otimes a_{i_{1}} \text { in } \stackrel{3}{\otimes} A_{i_{l}} \subset A .
$$

Then

$$
a^{3} \stackrel{\text { equi }}{=}\left(a_{i_{1}} \otimes a_{i_{2}} \otimes a_{i_{3}}\right)^{3} \stackrel{\text { equi }}{=} a_{i_{1}}^{3} a_{i_{2}}^{3} a_{i_{1}}^{3},
$$

in the minimal free summand of $A$ containing $a$, but

$$
\begin{aligned}
& a^{(3)}=\left(a_{i_{1}} a_{i_{2}} a_{i_{1}}\right)^{(3)} \\
& =a_{i_{1}} a_{i_{2}} a_{i_{1}} a_{i_{1}} a_{i_{2}} a_{i_{1}} a_{i_{1}} a_{i_{2}} a_{i_{1}} \text { (non-reduced word) } \\
& =a_{i_{1}} a_{i_{2}} a_{i_{1}}^{2} a_{i_{2}} a_{i_{1}}^{2} a_{i_{2}} a_{i_{1}} \quad \text { (reduced word) }
\end{aligned}
$$

i.e.,

$$
a^{(3)}=a_{i_{1}} a_{i_{2}} a_{i_{1}}^{2} a_{i_{2}} a_{i_{1}}^{2} a_{i_{2}} a_{i_{1}}
$$

is a free reduced word in $A$.

So, in the text below, if we use the term " $a^{k}$ " for a fixed free reduced word $a$, then it is in the sense of (8.1.3) contained in the minimal free summand of $A$ containing $a$; meanwhile, if we use the term “ $a^{(k)}$," then it means a free (non-reduced) word in $A$.

Note that $a^{k}=a^{(k)}$, only if $a$ is a free reduced word $a_{i_{1}}$ with its length- 1 in $A$, and hence, its minimal free summand of $A$ is identical to the free block $A_{i_{1}}$ in $A$, for all $k \in \mathbb{N}$.
Similar to $a^{k}$ and $a^{(k)}$, one can understand the adjoints $a^{*}$ and $a^{(*)}$ of a fixed free reduced word $a$ in $A$ as follows;

$$
a^{*} \stackrel{\text { equi }}{=}\left(\underset{\otimes_{l=1}}{\otimes} a_{i_{l}}\right)^{*}=\stackrel{n}{\otimes} a_{i_{l}}^{*} \stackrel{\text { equi }}{=} \prod_{l=1}^{n} a_{i_{l}}^{*},
$$

in the minimal free summand of $A$ containing $a$, but

$$
\begin{aligned}
a^{(*)} & =\left(\prod_{l=1}^{n} a_{i_{l}}\right)^{(*)}=\left(a_{i_{1}} a_{i_{2}} \ldots a_{i_{n}}\right)^{(*)} \\
& =a_{i_{n}}^{*} a_{i_{n-1}}^{*} \ldots a_{i_{2}}^{*} a_{i_{1}}^{*}=\prod_{l=1}^{n} a_{i_{n-l+1}}^{*},
\end{aligned}
$$

in $A$.

The free product linear functional $\varphi$ on $A$ satisfies that, whenever $a$ is a reduced free word in $A$ of (8.1.2), then

$$
\varphi\left(a^{k}\right)=\varphi\left(\prod_{l=1}^{n} a_{i_{l}}^{k}\right)=\prod_{l=1}^{n}\left(\varphi_{i_{l}}\left(a_{i_{l}}^{k}\right)\right),
$$

in the minimal free summand of $A$ containing $a$, by (8.1.3), for all $k \in \mathbb{N}$. Sometimes, by abusing (8.1.3), one can / may write

$$
\varphi\left(a^{k}\right)^{\text {equi }}=\varphi\left(\underset{l=1}{\otimes} a_{i_{l}}^{k}\right)=\prod_{l=1}^{n} \varphi\left(a_{i_{l}}^{k}\right),
$$

for all $k \in \mathbb{N}$.

Note that, in general,

$$
\varphi\left(a^{k}\right) \neq \varphi\left(a^{(k)}\right) \text {, for } k \in \mathbb{N} .
$$

However, the equality holds, if $a$ is a free reduced word with its length- 1 .

Now, let

$$
b=\sum_{l=1}^{n} b_{i_{l}} \in(A, \varphi) .
$$

We say that such an element $\mathrm{b}$ is a free sum in $A$, if all summands $b_{i_{1}}, \ldots, b_{i_{n}}$ of $b$ are contained in "mutually-distinct" minimal free summands of $A$ containing them as free reduced words. Then, similar to the above terminology, one can realize

$$
b \stackrel{\text { equi }}{=} \underset{l=1}{\otimes} b_{i_{l}}
$$

in the direct sum of the minimal free summands of $A$ containing $b_{i_{l}}$, for all $l=1, \ldots, n$ (also, called the minimal free summand of A containing the free sum $b$ ). So, it satisfies

$$
\begin{array}{r}
\varphi\left(b^{k}\right) \stackrel{\text { equi }}{=} \varphi\left(\left(\begin{array}{c}
n \\
\otimes \\
l=1
\end{array} b_{i_{l}}\right)^{k}\right)=\varphi\left(\begin{array}{c}
n \\
\otimes \\
l=1
\end{array} b_{i_{l}}^{k}\right) \\
\quad \text { equi } \\
\quad=\left(\sum_{l=1}^{n} b_{i_{l}}^{k}\right)=\sum_{l=1}^{n} \varphi\left(b_{i_{l}}^{k}\right),
\end{array}
$$


for all $k \in \mathbb{N}$. Here, each summand $\varphi\left(b_{i_{l}}^{k}\right)$ of (8.1.6) satisfies (8.1.4).

Notation and Remark 8.2. (From below, NR 8.2) Similar to NR 8.1, if b is a free sum in the sense of (8.1.5), then one can consider

$$
b^{(k)}=\left(\sum_{l=1}^{n} b_{i_{l}}\right)^{(k)}=\sum_{\left(i_{1}, \ldots, i_{k}\right) \in(1, \ldots, n]^{k}}\left(b_{i_{1}} b_{i_{l_{2}}} \ldots b_{i_{l}}\right),
$$

where the summands of $b^{(k)}$ are free (non-reduced) words in $A$.

For more about (free-probabilistic) free product algebras, and corresponding free probability spaces, see $[11,12,14]$ and cited papers therein.

\subsection{Free Product $C^{*}$-Probability Space $\left(\mathfrak{L} \mathfrak{S}, \tau^{0}\right)$}

In this section, we will use the same concepts and notations introduced in Section 8.1.

By (8.0.1), we have the family

$$
\left\{\mathfrak{L S}_{p}(j)=\left(\mathfrak{L} \mathfrak{S}_{p}, \tau_{p, j}^{0}\right): p \in \mathcal{P}, j \in \mathbb{Z}\right\}
$$

of Banach *-probability spaces.

Thus, one can define the free product Banach *-probability space,

$$
\mathfrak{L S}^{\text {denote }}=\left(\mathfrak{L S}, \tau^{0}\right)^{\text {def }} \underset{p \in \mathcal{P}, j \in \mathbb{Z}}{\star}\left(\mathfrak{L S}_{p}, \tau_{p, j}^{0}\right),(8.2 .1)
$$

as in Section 8.1.

Definition 8.1. The Banach *-probability space $\mathfrak{L S}=\left(\mathfrak{L S}, \tau^{0}\right)$ of (8.2.1) is called the radial-Adelic (Banach-*-) probability space. If we understand $\mathfrak{L} \mathfrak{S}$ as a Banach *-algebra, we call it the radial-Adelic (Banach *-) algebra.

Let $\mathfrak{L S}$ be the radial-Adelic probability space (8.2.1). Then, we obtain a subset

$$
\left\{Q_{p, j}=l_{p} \otimes P_{p, j} \in \mathfrak{L} \mathfrak{S}_{p}(j)\right\}_{p \in \mathcal{P}, j \in \mathbb{Z}}
$$

of $\mathfrak{L} \mathfrak{S}$, consisting of $p^{2(j+1)}$-semicircular elements $Q_{p, j}$ in the free blocks $\mathfrak{L S}_{p}(j)$ of $\mathfrak{L} \mathfrak{S}$. Remark here that, by the choice of $Q_{p, j}$ in the family, all $Q_{p, j}$ are taken from the mutually-distinct free blocks $\mathfrak{L S}_{p}(j)$ of $\mathfrak{L S}$. It means that all elements $Q_{p, j}$ are mutually-free from each other in $\mathfrak{L} \mathfrak{S}$.

Recall that a subset $S=\left\{a_{t}\right\}_{t \in \Delta}$ of an arbitrary *-probability space $(A, \varphi)$ is said to be a free family, if, for all pairs $\left(t_{1}, t_{2}\right) \in \Delta^{2}$ of "distinct” elements $t_{1}$ and $t_{2}$ of $\Delta$, the corresponding operators $a_{t_{1}}$ and $a_{t_{2}}$ are free in $(A, \varphi)$ (e.g., [12,14]).
Definition 8.2. Let $S=\left\{a_{t}\right\}_{t \in \Delta}$ be a free family in a *-probability space $(A, \varphi)$. This family $S$ is said to be a free semicircular family, if every element $a_{t}$ of $S$ is semicircular, for all $t \in \Delta$. Similarly, the family $S$ is called a free weighted-semicircular family, if all elements $a_{t}$ of $S$ are weighted-semicircular, for all $t \in \Delta$.

So, by the very construction (8.2.1) of our radial-Adelic probability space $\mathfrak{L} \mathfrak{S}$, we obtain the following fact.

Theorem 8.1. Let $\mathfrak{L} \mathfrak{S}$ be the radial-Adelic probability space (8.2.1). And let $Q_{p, j}$ be the generating operators $l_{p} \otimes P_{p, j}$ of $\mathfrak{L} \mathfrak{S}$.

(8.2.2) A family $\left\{Q_{p, j} \in \mathfrak{L} \mathfrak{S}_{p}(j)\right\}_{j \in \mathbb{Z}}$ is a weightedsemicircular free family in $\mathfrak{L S}$, for a fixed $p \in \mathcal{P}$.

(8.2.3) A family $\left\{Q_{p, j} \in \mathfrak{L S}_{p}(j)\right\}_{p \in \mathcal{P}}$ is a weightedsemicircular free family in $\mathfrak{L} \mathcal{S}$, for a fixed $j \in \mathbb{Z}$.

(8.2.4) A family $\left\{Q_{p, j} \in \mathfrak{L} \mathfrak{S}_{p}(j)\right\}_{p \in \mathcal{P}, j \in \mathbb{Z}}$ is a weighted-semicircular free family in $\mathfrak{L} \mathfrak{S}$.

Proof. First, let's take a family $\left\{Q_{p, j} \in \mathfrak{L S}_{p}(j)\right\}_{j \in \mathbb{Z}}$, by fixing a prime $p$. Then one can understand this family is taken from

$$
\mathfrak{L S}_{p}(\mathbb{Z}) \stackrel{\text { denote }}{=} \underset{j \in \mathbb{Z}}{\star} \mathfrak{L} \mathfrak{S}_{p}(j) \text { in } \mathfrak{L} \mathfrak{S},
$$

which forms a free block of $\mathfrak{L} \mathfrak{S}$, because

$$
\mathfrak{L S}=\underset{p \in \mathcal{P}}{\star} \mathfrak{L} \mathfrak{S}_{p}(\mathbb{Z}),
$$

by (8.2.1). Therefore, the subset

$$
\left\{Q_{p, j} \in \mathfrak{L S}_{p}(j)\right\}_{j \in \mathbb{Z}}
$$

is a free family in $\mathfrak{L S}_{p}(\mathbb{Z})$ for the fixed prime $p$. So, the family $\left\{Q_{p, j}\right\}_{j \in \mathbb{Z}}$ is also a free family in $\mathfrak{L} \mathfrak{S}$.

Now, assume we have a family $\left\{Q_{q, j} \in \mathfrak{L S}_{q}(j)\right\}_{q \in \mathcal{P}}$ in $\mathfrak{L} \mathfrak{S}$, for a fixed integer $j \in \mathbb{Z}$. Then, this family is a subset of

$$
\mathfrak{L S}_{\mathcal{P}}(j) \stackrel{\text { denote }}{=} \underset{p \in \mathcal{P}}{\star} \mathfrak{L} \mathfrak{S}_{p}(j) \text { of } \mathfrak{L} \mathfrak{S},
$$

because

$$
\mathfrak{L S}=\underset{j \in \mathbb{Z}}{\star} \mathfrak{L} \mathfrak{S}_{\mathcal{P}}(j)
$$

by (8.2.1). Thus, the subset

$$
\left\{Q_{q, j} \in \mathfrak{L} \mathfrak{S}_{q}(j)\right\}_{q \in \mathcal{P}}
$$

forms a free family in $\mathfrak{L S}_{q}(j)$ for the fixed integer $j$. Therefore, the family $\left\{Q_{q, j}\right\}_{q \in \mathcal{P}}$ is a free family in $\mathfrak{L} \mathfrak{S}$. 
Finally, consider the family $\left\{Q_{p, j} \in \mathcal{L} \mathfrak{S}_{p}(j)\right\}_{p \in \mathcal{P}, j \in \mathbb{Z}}$. We re-write this family by

$$
\bigcup_{j \in \mathbb{Z}}\left\{Q_{p, j}\right\}_{p \in \mathcal{P}}
$$

Each family $\left\{Q_{p, j}\right\}_{p \in \mathcal{P}}$ is a free family in $\mathfrak{L S}_{\mathcal{P}}(j)$ for each $j \in \mathbb{Z}$. Note that the family $\left\{\mathfrak{L S}_{\mathcal{P}}(j)\right\}_{j \in \mathbb{Z}}$ forms a free family, and hence, the sub-families $\left\{Q_{p, j}\right\}_{p \in \mathcal{P}}$ is a free family in $\mathfrak{L S}$, too.

Therefore, the family

$$
\left\{Q_{p, j} \in \mathcal{L} \mathfrak{S}_{p}(j)\right\}_{p \in \mathcal{P}, j \in \mathbb{Z}}
$$

is a free family in $\mathfrak{L} \mathfrak{S}$.

Recall that all elements $Q_{p, j}$ are $p^{2(j+1)}$-semicircular in $\mathfrak{L S}_{p}(j)$, for all $p \in \mathcal{P}$ and $j \in \mathbb{Z}$, and hence, they are weighted-semicircular in $\mathfrak{L} \mathfrak{S}$. Therefore, the statements (8.2.2), (8.2.3) and (8.2.4) hold.

\subsection{Semicircular Elements Induced by $\left\{Q_{p, j}\right\}_{p \in \mathcal{P}, j \in \mathbb{Z}}$}

Let $\mathfrak{L} \mathfrak{S}=(\mathfrak{L S}, \tau)$ be the radial Adelic probability space (8.2.1). Define the elements $\Theta_{p, j}$ of $\mathfrak{L S}$ by

$$
\Theta_{p, j} \stackrel{\text { def }}{=} \frac{1}{p^{j+1}} Q_{p, j}, \text { for all } p \in \mathcal{P}, j \in \mathbb{Z} .
$$

Then, by the self-adjointness of $Q_{p, j}$, the operators $\Theta_{p, j}$ of (8.3.1) are self-adjoint in $\mathfrak{L} \mathfrak{S}$.

Also, one obtains the following free-cumulant computation; if $k_{n}^{0}(\ldots)$ is the free cumulant on $\mathfrak{L} \mathfrak{S}$ with respect to the linear functional $\tau^{0}$ on the radial-Adelic algebra $\mathfrak{L} \mathfrak{S}$, then

$$
\begin{aligned}
& k_{n}^{0}\left(\Theta_{p, j}, \ldots, \Theta_{p, j}\right) \\
& =k_{n}^{0, p, j}\left(\frac{1}{p^{j+1}} Q_{p, j}, \ldots, \frac{1}{p^{j+1}} Q_{p, j}\right) \\
& =\left(\frac{1}{p^{j+1}}\right)^{n} k_{n}^{0, p, j}\left(Q_{p, j}, \ldots, Q_{p, j}\right),
\end{aligned}
$$

by the bimodule-map property of free cumulant (e.g., [12]), for all $n \in \mathbb{N}$, where $k_{n}^{0, p, j}(\ldots)$ are the free cumulants on the free blocks $\mathfrak{L S}_{p}(j)$ of $\mathfrak{L} \mathfrak{S}$, in terms of the linear functional $\tau_{p, j}^{0}$ on $\mathfrak{L} \mathfrak{S}_{p}$, for all $p \in \mathcal{P}$, $j \in \mathbb{Z}$.

By (8.3.2), we obtain the following result.

Theorem 8.2. Let $\Theta_{p, j}=\frac{1}{p^{j+1}} Q_{p, j}$ be free random variables (8.3.1) of the radial-Adelic probability space $\mathfrak{L S}=\left(\mathfrak{L S}, \tau^{0}\right)$, for $p \in \mathcal{P}, j \in \mathbb{Z}$. Then $\Theta_{p, j}$ are semicircular elements, and the families

$$
\begin{aligned}
& \Theta(p)=\left\{\Theta_{p, j} \in \mathcal{L S}_{p}(j): j \in \mathbb{Z}\right\}, \\
& \Theta(j)=\left\{\Theta_{p, j} \in \mathcal{L} \mathcal{S}_{p}(j): p \in \mathcal{P}\right\},
\end{aligned}
$$

and

$$
\Theta=\left\{\Theta_{p, j} \in \mathfrak{L S}_{p}(j): p \in \mathcal{P}, j \in \mathbb{Z}\right\}
$$

form free semicircular families in $\mathfrak{L} \mathfrak{S}$.

Proof. Consider that

$$
k_{n}^{0}\left(\Theta_{p, j}, \ldots, \Theta_{p, j}\right)=\left(\frac{1}{p^{j+1}}\right)^{n} k_{n}^{0, p, j}\left(Q_{p, j}, \ldots, Q_{p, j}\right)
$$

by (8.3.2)

$$
= \begin{cases}\left(\frac{1}{p^{j+1}}\right)^{2} k_{2}^{0, p, j}\left(Q_{p, j}, Q_{p, j}\right) & \text { if } n=2 \\ \left(\frac{1}{p^{j+1}}\right)^{n} k_{n}^{0, p, j}\left(Q_{p, j}, \ldots, Q_{p, j}\right)=0 & \text { otherwise, }\end{cases}
$$

by the $p^{2(j+1)}$-semicircularity of $Q_{p, j}$ in $\mathfrak{L} \mathfrak{S}$

$$
\begin{aligned}
& = \begin{cases}\left(\frac{1}{p^{j+1}}\right)^{2}\left(p^{j+1}\right)^{2} & \text { if } n=2 \\
0 & \text { otherwise, }\end{cases} \\
& = \begin{cases}1 & \text { if } n=2 \\
0 & \text { otherwise, }\end{cases}
\end{aligned}
$$

for all $n \in \mathbb{N}$.

By the free-cumulant computation (8.3.4), the selfadjoint operators $\Theta_{p, j}$ are semicircular in $\mathfrak{L} \mathfrak{S}$, by (6.1.3), for all $p \in \mathcal{P}, j \in \mathbb{Z}$.

Thus, the families $\Theta(p), \Theta(j)$ and $\Theta$ of (8.3.3) form free families in $\mathfrak{L S}$ by (8.2.2), (8.2.3) and (8.2.4), respectively, because all elements $\Theta_{p, j}$ are simply scalar multiples of $Q_{p, j}$ contained in mutually-distinct free blocks $\mathfrak{L S}_{p}(j)$ of $\mathfrak{L} \mathfrak{S}$, for all $j \in \mathbb{Z}, \quad p \in \mathcal{P}$. Therefore, by (8.3.4), the families of (8.3.3) are free semicircular families in $\mathfrak{L} \mathfrak{S}$.

\section{Operators of $\mathfrak{L} \mathfrak{S}$ Generated by $\Theta$}

Let $\mathfrak{L} \mathfrak{S}=\left(\mathfrak{L} \mathfrak{S}, \tau^{0}\right)$ be the radial-Adelic probability space in the sense of (8.2.1), and let

$$
\Theta=\left(\Theta_{p, j} \in \mathfrak{L S}_{p}(j): p \in \mathcal{P}, j \in \mathbb{Z}\right)
$$

be the free semicircular family in (8.3.3), where 
$\mathfrak{L S}_{p}(j)=\left(\mathfrak{L S}_{p}, \tau_{p, j}^{0}\right)$ are the free blocks of $\mathfrak{L} \mathfrak{S}$, for all $p \in \mathcal{P}, j \in \mathbb{Z}$. Throughout this section, fix $N \in \mathbb{N} \backslash\{1\}$, and we consider certain free-distributional data of operators generated by the free semicircular family $\Theta$,

$$
M_{p_{1}, \ldots, p_{N}}^{j_{1}, \ldots, j_{N}: n_{1}, \ldots, n_{N}}=\prod_{l=1}^{N} \Theta_{p_{l}, j_{l}}^{n_{l}}
$$

in $\mathfrak{L} \mathfrak{S}=\left(\mathfrak{L} \mathfrak{S}, \tau^{0}\right)$, for

$$
j_{1}, \ldots, j_{N} \in \mathbb{Z} \text {, and } p_{1}, \ldots, p_{N} \in \mathcal{P} \text {, }
$$

and

$$
n_{1}, \ldots, n_{N} \in \mathbb{N} .
$$

Theorem 9.1. Let $M_{p_{1}, \ldots, p_{N}}^{j_{1}, \ldots, j_{N}: n_{1}, \ldots, n_{N}}$ be an operator (9.1) generated by the free semicircular family $\Theta$ of (8.3.3) in the radial-Adelic probability space $\mathfrak{L} \mathfrak{S}$. If either the prime-sequence $\left(p_{1}, \ldots, p_{N}\right)$, or the integer-sequence $\left(j_{1}, \ldots, j_{N}\right)$ is an alternating sequence, then

$$
\tau^{0}\left(\left(M_{p_{1}, \ldots, p_{N}}^{j_{1}, \ldots, j_{N}: n_{1}, \ldots, n_{N}}\right)^{k}\right)=\prod_{l=1}^{N} \omega_{k n_{l}} \frac{c_{k n_{l}}}{2},
$$

in the minimal free summand of $\mathfrak{L} \mathfrak{S}$ containing $M_{p_{1}, \ldots, p_{N}}^{j_{1}, \ldots, j_{N}: n_{1}, \ldots, n_{N}}$.

Under the same hypothesis, if either $p_{N} \neq p_{1}$ in $\mathcal{P}$, or $j_{N} \neq j_{1}$ in $\mathbb{Z}$, then

$$
\begin{aligned}
& \tau^{0}\left(\left(M_{p_{1}, \ldots, p_{N}}^{j_{1}, \ldots, j_{N}: n_{1}, \ldots, n_{N}}\right)^{(k)}\right)=\prod_{l=1}^{N} \omega_{k n_{l}}\left(c_{\frac{k n_{l}}{2}}\right)^{k} \\
& =\tau^{0}\left(\left(\left(M_{p_{1}, \ldots, p_{N}}^{j_{1}, \ldots, j_{N}: n_{1}, \ldots, n_{N}}\right)^{(*)}\right)^{(k)}\right)
\end{aligned}
$$

in $\mathfrak{L S}$, for all $k \in \mathbb{N}$.

Proof. Let $T=M_{p_{1}, \ldots, p_{N}}^{j_{1}, \ldots, j_{N}: n_{1}, \ldots, n_{N}}$ be in the sense of (9.1) in $\mathfrak{L} \mathfrak{S}$. Assume either the prime-sequence $\left(p_{1}, \ldots, p_{N}\right)$, or the integer-sequence $\left(j_{1}, \ldots, j_{N}\right)$ is an alternating sequence. Then this operator $T$ is a free reduced word in $\mathfrak{L S}$, and hence,

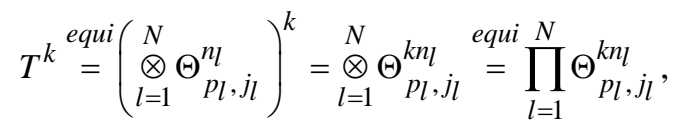

in the minimal free summand of $\mathfrak{L S}$ containing $T$, by (8.1.3), for all $k \in \mathbb{N}$.

Therefore, one has that

$$
\tau^{0}\left(T^{k}\right)=\prod_{l=1}^{N} \tau_{p_{l}, j_{l}}^{0}\left(\Theta_{p_{l}, j_{l}}^{k n_{l}}\right)=\prod_{l=1}^{N} \omega_{k n_{l}} c_{\frac{k n_{l}}{2}},
$$

in the free block, by the semicircularity of $\Theta_{p_{l}, j_{l}}$.

Now, let $T$ be given as above in $\mathfrak{L} \mathfrak{S}$. Suppose either $\left(p_{1}, \ldots, p_{N}\right)$, or $\left(j_{1}, \ldots, j_{N}\right)$ is alternating in $\mathcal{P}$, respectively, in $\mathbb{Z}$, and assume further that

$$
p_{N} \neq p_{1} \text { in } \mathcal{P} \text {, or } j_{N} \neq j_{1} \text { in } \mathbb{Z} \text {. }
$$

Then this operator $T$ is not only a free reduced word in $\mathfrak{L S}$, but also $T^{(k)}$ are free reduced words in $\mathfrak{L} \mathfrak{S}$, satisfying

$$
\begin{aligned}
& T^{(k)}=\left(\prod_{l=1}^{N} \Theta_{p_{l}, j_{l}}^{n_{l}}\right)^{(k)} \\
& =\left(\prod_{l=1}^{N} \Theta_{p_{l}, j_{l}}^{n_{l}}\right)\left(\prod_{l=1}^{N} \Theta_{p_{l}, j_{l}}^{n_{l}}\right) \ldots\left(\prod_{l=1}^{N} \Theta_{p_{l}, j_{l}}^{n_{l}}\right),
\end{aligned}
$$

in $\mathfrak{L} \mathfrak{S}$, for all $k \in \mathbb{N}$. Therefore, we have

$$
\tau^{0}\left(T^{(k)}\right)=\left(\prod_{l=1}^{N} \tau_{p_{l}, j_{l}}^{0}\left(\Theta_{p_{l}, j_{l}}^{k n_{l}}\right)\right)^{k}=\prod_{l=1}^{N} \omega_{k n_{l}}\left(c_{k n_{l}}\right)^{k},
$$

for all $k \in \mathbb{N}$, by the semicircularity of $\Theta_{p_{l}, j_{l}}$.

Now, remark that

$$
T^{(*)}=\left(\prod_{l=1}^{N} \Theta_{p_{l}, j_{l}}^{n_{l}}\right)^{(*)}=\prod_{l=0}^{N-1} \Theta_{p_{N-l}, j_{N-l}}^{n_{N-l}}
$$

in $\mathfrak{L} \mathfrak{S}$, by the self-adjointness of our semicircular elements $\Theta_{p_{l}, j_{l}}$, for all $l=1, \ldots, N$. So,

$$
\left(T^{(*)}\right)^{(k)}=\left(\prod_{l=0}^{N-1} \Theta_{p_{N-l}, j_{N-l}}^{n_{N-l}}\right)^{(k)},
$$

for all $k \in \mathbb{N}$, moreover, it forms a free reduced word in $\mathfrak{L} \mathfrak{S}$, too, because either

$$
\left(p_{N}, \ldots, p_{2}, p_{1}\right) \text { or }\left(j_{N}, \ldots, j_{2}, j_{1}\right)
$$

is alternating in $\mathcal{P}$, respectively, in $\mathbb{Z}$. Also, since either

$$
p_{1} \neq p_{N} \text { in } \mathcal{P} \text {, or } j_{1} \neq j_{N} \text { in } \mathbb{Z} \text {, }
$$

the operators $\left(T^{(*)}\right)^{(k)}$ are free reduced words in $\mathfrak{L} \mathfrak{S}$, too, for all $k \in \mathbb{N}$.

And hence, similar to the above case

$$
\tau^{0}\left(\left(T^{(*)}\right)^{(k)}\right)=\left(\tau^{0}\left(T^{(*)}\right)\right)^{k}=\left(\tau^{0}(T)\right)^{k},
$$

for all $k \in \mathbb{N}$.

Therefore, the free-distributional data (9.3) holds.

The formulas (9.2) and (9.3) characterize the free distributions of free reduced words $M_{p_{1}, \ldots, p_{N}}^{j_{1}, \ldots, j_{N}: n_{1}, \ldots, n_{N}}$, where either $p_{1} \neq p_{N}$ in $\mathcal{P}$, or $j_{1} \neq j_{N}$ in $\mathbb{Z}$. 
Let $(A, \varphi)$ and $(B, \psi)$ be arbitrary topological *-probability spaces, and let $a \in(A, \varphi)$ and $\mathrm{b} b \in(B, \psi)$ be "self-adjoint." These operators $a$ and $b$ are said to be identically free-distributed, if

$$
\varphi\left(a^{n}\right)=\psi\left(b^{n}\right), \text { for all } n \in \mathbb{N} .
$$

Motivated by (9.4), one can obtain the following universalized result.

Theorem 9.2. Let $(A, \varphi)$ be an arbitrary topological *-probability space, and let $\Xi=\left\{s_{l}\right\}_{l=1}^{N}$ be a free semicircular family in $A$, for some $N \in \mathbb{N}$. Let

$$
M=\prod_{l=1}^{N} s_{l}^{n_{l}}, \text { with } s_{1}, \ldots, s_{N} \in \Xi,
$$

where $n_{1}, \ldots, n_{N} \in \mathbb{N}$, and $s_{1}, \ldots, s_{N}$ are mutually distinct in $\Xi$. Then

$$
\varphi\left(M^{k}\right)=\prod_{l=1}^{N} \omega_{k n_{l}} c_{\frac{k n_{l}}{2}},
$$

$$
N
$$

in the minimal free summand $\otimes_{\mathbb{C}} A_{l}$ of $A$ containing $M$; for all $k \in \mathbb{N}$,

Also, if $M$ is as above in $(A, \varphi)$, and if $s_{1} \neq s_{N}$ in $\Xi$, then

$$
\varphi\left(M^{(k)}\right)=\prod_{l=1}^{N} \omega_{k n_{l}}\left(\frac{c_{k n_{l}}}{2}\right)^{k}, \text { for all } k \in \mathbb{N} .
$$

Proof. Since the family $\Xi$ is assumed to be a free semicircular family, all elements $s_{1}, \ldots, s_{N}$ are not only free from each other in $(A, \varphi)$, but also semicircular in $(A, \varphi)$. By the definitions of $M$, the operator $M$ is a free reduced word in $A$, satisfying the equivalences,

$$
M \stackrel{\text { equi }}{=} \underset{l=1}{\otimes} s_{l}^{n_{l}}
$$

in the minimal free summand of $A$ containing $M$.

Therefore, by the identically-free-distributedness (9.4), one can obtain the free-distributional data (9.5) in the minimal free summand $\otimes_{\mathbb{C}}^{N} A_{l}$ of $A$ containing $M$, where $l=1$

$A_{l}$ are the *-subalgebras of $\mathrm{A}$ generated by $s_{l}$, for all $l=1, \ldots, N$.

Similar to (9.5), we obtain the free-distributional data (9.6) by (9.3), because $M^{(k)}$ form free reduced words in $(A, \varphi)$, for all $k \in \mathbb{N}$.

In the proof of (9.5) and (9.6), we use the identically free-distributedness of the semicircular law.

Remark 9.1. Our main results (9.2) and (9.3) show that the free distributions of operators (9.1) in $\mathfrak{L S}$ generated by our free semicircular family $\Theta$ of (8.3.3) are determined universally, up to the identically-free-distributedness of semicircularity, by (9.5) and (9.6). It demonstrates that we lost some interesting "local" free-probabilistic information came from our number-theoretic settings from p-adic, and Adelic analysis. So, we escape from such a universalization in Section 10 below.

\section{Operators of $\mathfrak{L} \mathfrak{S}$ Generated by $\mathcal{Q}$}

In this section, we use same concepts and notations introduced in Notation 8.1, NR 8.1, and NR 8.2. Throughout this section, we fix $N \in \mathbb{N} \backslash\{1\}$, and let

$$
p_{1}, \ldots, p_{N} \in \mathcal{P} \text {, and } j_{1}, \ldots, j_{N} \in \mathbb{Z},
$$

and

$$
n_{1}, \ldots, n_{N} \in \mathbb{N}
$$

Let $\mathcal{Q}$ be the weighted-semicircular free family (8.2.4) of $\mathfrak{L S}$, i.e.,

$$
\mathcal{Q} \stackrel{\text { def }}{=}\left\{Q_{p, j} \in \mathfrak{L S}_{p}(j): j \in \mathbb{Z}, p \in \mathcal{P}\right\},
$$

consisting of $p^{2(j+1)}$-semicircular elements

$$
Q_{p, j}=l_{p} \otimes P_{p, j} \in \mathcal{L} \mathfrak{S}_{p}(j)=\left(\mathfrak{L} \mathfrak{S}_{p}, \tau_{p, j}^{0}\right),
$$

which are free from each other in the radial-Adelic probability space $\mathfrak{L} \mathfrak{S}=\left(\mathfrak{L} \mathfrak{S}, \tau^{0}\right)$, for all $j \in \mathbb{Z}, p \in \mathcal{P}$.

Define operators $X_{p_{1}, \ldots, p_{N}}^{j_{1}, \ldots, j_{N}: n_{1}, \ldots, n_{N}}$ of $\mathfrak{L} \mathfrak{S}$ by the operators, induced by the free family $\mathcal{Q}$ of $(10,1)$, by

$$
X_{p_{1}, \ldots, p_{N}}^{j_{1}, \ldots, j_{N}: n_{1}, \ldots, n_{N}}=\prod_{k=1}^{N} Q_{p_{k}, j_{k}}^{n_{k}},
$$

in $\mathfrak{L} \mathfrak{S}$.

Theorem 10.1. Let $X_{p_{1}, \ldots, p_{N}}^{j_{1}, \ldots, j_{N}: n_{1}, \ldots, n_{N}}$ be an operator of (10.2), generated by the free weighted-semicircular family $\mathcal{Q}$ of (10.1), in the radial-Adelic probability space $\mathfrak{L} \mathfrak{S}$. Assume that either the prime-sequence $\left(p_{1}, \ldots, p_{N}\right)$, or the integer-sequence $\left(j_{1}, \ldots, j_{N}\right)$ is alternating in $\mathcal{P}$, respectively, in $\mathbb{Z}$. Then, in the minimal free summand of $\mathfrak{L S}$ containing it, we have

$\tau^{0}\left(\left(X_{p_{1}, \ldots, p_{N}}^{j_{1}, \ldots, j_{N}: n_{1}, \ldots, n_{N}}\right)^{k}\right)=\prod_{l=1}^{N} \omega_{k n_{l}}\left(p_{l}^{j_{l}+1}\right)^{k n_{l}} c_{\frac{k n_{l}}{2}}$,

for all $k \in \mathbb{N}$.

Proof. Let $X_{p_{1}, \ldots, p_{N}}^{j_{1}, \ldots, j_{N}: n_{1}, \ldots, n_{N}}$ be in the sense of (10.2) in $\mathfrak{L S}$, and assume either

$$
\left(p_{1}, \ldots, p_{N}\right), \text { or }\left(j_{1}, \ldots, j_{N}\right)
$$

is an alternating sequence in $\mathcal{P}$, respectively, in $\mathbb{Z}$. 
Then this operator $X$ is a free reduced word in $\mathfrak{L} \mathfrak{S}$. So, it satisfies that

$$
\begin{aligned}
& X^{k} \stackrel{\text { equi }}{=}\left(\underset{l=1}{\otimes} Q_{p_{l}, j_{l}}^{n_{l}}\right)^{k}=\underset{l=1}{\otimes}\left(Q_{p_{l}, j_{l}}^{n_{l}}\right)^{k} \\
& =\underset{l=1}{\otimes} Q_{p_{l}, j_{l}}^{k n_{l}} \stackrel{\text { equi }}{=} \prod_{l=1}^{N} Q_{p_{l}, j_{l}}^{k n_{l}},
\end{aligned}
$$

in the minimal free summand of $\mathfrak{L S}$ containing $X$, for all $k \in \mathbb{N}$.

By the equivalence (10.4), one has that

$$
\begin{aligned}
& \tau^{0}\left(X^{k}\right)=\tau^{0}\left(\prod_{l=1}^{N} Q_{p_{l}, j_{l}}^{k n_{l}}\right) \stackrel{\text { equi }}{=} \prod_{l=1}^{N} \tau_{p_{l}, j_{l}}^{0}\left(Q_{p_{l}, j_{l}}^{k n_{l}}\right) \\
& =\prod_{l=1}^{N} \omega_{k n_{l}}\left(p_{l}^{\left(j_{l}+1\right)}\right)^{k n_{l}} \frac{c_{\frac{k n_{l}}{2}}}{2}
\end{aligned}
$$

for all $k \in \mathbb{N}$, by the $p_{l}^{2\left(j_{l}+1\right)}$-semicircularity of $Q_{p_{l}, j_{l}}$, for all $l=1, \ldots, N$.

Therefore, the free-moment formula (10.3) holds in the minimal free summand of $\mathfrak{L S}$ containing $X$.

Remark that, by the equivalence (10.4), one can check the self-adjointness of the operator $X_{p_{1}, \ldots, p_{N}}^{j_{1}, \ldots, j_{N}: n_{1}, \ldots, n_{N}}$ in the minimal free summand of $\mathfrak{L S}$ containing it, i.e.,

$$
\left(\begin{array}{l}
N \\
l_{l=1}
\end{array} Q_{p_{l}, j_{l}}^{n_{l}}\right)^{*}=\underset{l=1}{\otimes}\left(Q_{p_{l}, j_{l}}^{n_{l}}\right)^{*}=\underset{l=1}{\otimes} Q_{p_{l}, j_{l}}^{n_{l}},
$$

by the self-adjointness of $Q_{p_{l}, j_{l}}^{n_{l}}$, for all $l=1, \ldots, N$. This self-adjointness guarantees that the free-moment formula (10.3) characterizes the free distribution of $X_{p_{1}, \ldots, p_{N}}^{j_{1}, \ldots, j_{N}: n_{1}, \ldots, n_{N}}$ in the minimal free summand of $\mathfrak{L} \mathfrak{S}$ containing it. (Remark that the above self-adjointness does not hold in $\mathfrak{L} \mathfrak{S}$ ! It holds "in the minimal free summand.")

Theorem 10.2. Let $X_{p_{1}, \ldots, p_{N}}^{j_{1}, \ldots, j_{N}: n_{1}, \ldots, n_{N}}$ be an operator (10.2) in the radial-Adelic probability space $\mathfrak{L} \mathfrak{S}$. Assume that either $\left(p_{1}, \ldots, p_{N}\right)$, or $\left(j_{1}, \ldots, j_{N}\right)$ is alternating in $\mathcal{P}$, respectively, in $\mathbb{Z}$. If either

$$
p_{N} \neq p_{1} \text {, in } \mathcal{P} \text {, or } j_{N} \neq j_{1} \text { in } \mathbb{Z},
$$

then we have

$$
\begin{aligned}
& \tau^{0}\left(\left(X_{p_{1}, \ldots, p_{N}}^{j_{1}, \ldots, j_{N}: n_{1}, \ldots, n_{N}}\right)^{(k)}\right) \\
& =\sum_{l=1}^{N} \omega_{n_{l}}\left(\left(p_{l}^{j_{l}+1}\right)^{n_{l}} c_{\frac{n_{l}}{2}}\right)^{k} \\
& =\tau^{0}\left(\left(\left(X_{p_{1}, \ldots, p_{N}}^{j_{1}, \ldots, j_{N}: n_{1}, \ldots, n_{N}}\right)^{(*)}\right)^{(k)}\right),
\end{aligned}
$$

for all $k \in \mathbb{N}$.
Proof. Let $X_{p_{1}, \ldots, p_{N}}^{j_{1}, \ldots, j_{N}: n_{1}, \ldots, n_{N}}$ be in the sense of (10.2) in $\mathfrak{L} \mathfrak{S}$, and suppose that either

$$
\left(p_{1}, \ldots, p_{N}\right) \text { is alternating in } \mathcal{P},
$$

or

$$
\left(j_{1}, \ldots, j_{N}\right) \text { is alternating in } \mathbb{Z} \text {. }
$$

Then the operator $X$ forms a free reduced word in $\mathfrak{L} \mathfrak{S}$. Also, suppose that either

$$
p_{N} \neq p_{1} \text {, in } \mathcal{P} \text {, or } j_{N} \neq j_{1} \text { in } \mathbb{Z} \text {. }
$$

Then the powers $X^{(k)}$ of $X$ form free reduced words "in $\mathfrak{L S}$," too, for all $k \in \mathbb{N}$. Thus, one can get that

$$
\begin{aligned}
& \tau^{0}\left(X^{(k)}\right)=\tau^{0}(\underbrace{X X \ldots . X}_{k \text {-times }}) \\
& =\tau^{0}\left(\left(\prod_{k=1}^{N} Q_{p_{k}, j_{k}}^{n_{k}}\right)\left(\prod_{k=1}^{N} Q_{p_{k}, j_{k}}^{n_{k}}\right) \ldots\left(\prod_{k=1}^{N} Q_{p_{k}, j_{k}}^{n_{k}}\right)\right) \\
& =\left(\prod_{k=1}^{N} \tau_{p_{k}, j_{k}}^{0}\left(Q_{p_{k}, j_{k}}^{n_{k}}\right)\right) \ldots\left(\prod_{k=1}^{N} \tau_{p_{k}, j_{k}}^{0}\left(Q_{p_{k}, j_{k}}^{n_{k}}\right)\right) \\
& =\left(\prod_{k=1}^{N} \tau_{p_{k}, j_{k}}^{0}\left(Q_{p_{k}, j_{k}}^{n_{k}}\right)\right)^{k}=\left(\tau^{0}(X)\right)^{k} \\
& =\left(\prod_{k=1}^{N} \omega_{n_{k}} p_{k}^{2\left(j_{k}+1\right)} c_{\frac{n_{k}}{2}}\right)^{k} \\
& =\prod_{k=1}^{N}\left(\omega_{n_{k}} p_{k}^{2\left(j_{k}+1\right)} c_{\frac{n_{k}}{2}}\right)^{k} \\
& =\prod_{k=1}^{N}\left(\omega_{n_{k}}\left(p_{k}^{2\left(j_{k}+1\right)} c_{\frac{n_{k}}{2}}\right)^{k},\right.
\end{aligned}
$$

for all $k \in \mathbb{N}$. So, the first equality of the formula (10.5) holds by (10.6) Note that $X$ is not self-adjoint in $\mathfrak{L S}$, because

$$
X^{(*)}=Q_{p_{N}, j_{N}}^{n_{N}} \ldots Q_{p_{2}, j_{2}}^{n_{2}} Q_{p_{1}, j_{1}}^{n_{1}} \text { in } \mathfrak{L} \mathfrak{S} .
$$

Thus, similar to (10.6), one obtains that

$$
\tau^{0}\left(\left(X^{(*)}\right)^{(k)}\right)=\left(\tau^{0}\left(X^{(*)}\right)\right)^{k}=\left(\tau^{0}(X)\right)^{k},
$$

for all $k \in \mathbb{N}$.

The formula (10.7) proves the second equality of (10.5) Therefore, by (10.6) and (10.7), the free-distributional data (10.5) holds.

The free-distributional data (10.5) not only gives free-probabilistic information of the operators (10.2), but also provides tools to compute the (joint) free distributions of operators (10.2) in the radial-Adelic probability space $\mathfrak{L S}$. 


\section{How Close Our Weighted-Semicircularity is to Semicircularity?}

In Sections 9 and 10, we studied free-distributional data of the free reduced words generated by our semicircular elements, and those generated by our weighted-semicircular elements. In particular, we considered such data both in minimal free summands of the radial-Adelic probability space $\mathfrak{L} \mathfrak{S}=\left(\mathfrak{L S}, \tau^{0}\right)$, and the Banach *-probability space $\mathfrak{L} \mathfrak{S}$, itself.

Let $\mathcal{Q}$ and $\Theta$ be subsets of $\mathfrak{L S}$ defined by

$$
\mathcal{Q}=\left\{Q_{p, j} \in \mathcal{L} \mathfrak{S}_{p}(j)\right\}_{j \in \mathbb{Z}, p \in \mathcal{P}},
$$

and

$$
\Theta=\left\{\Theta_{p, j}=\frac{1}{p^{j+1}} Q_{p, j} \in \mathfrak{L} \mathfrak{S}_{p}(j)\right\}_{j \in \mathbb{Z}, p \in \mathcal{P}} .
$$

Then the family $\mathcal{Q}$ of (11.1) is a free weighted-semicircular family (by (8.2.4)), and the family $\Theta$ of (11.1) is a free semicircular family (by (8.3.3)) in $\mathfrak{L} \mathfrak{S}$.

Let $W=\left(p_{1}, \ldots, p_{N}\right)$ be a finite sequence in $\mathcal{P}$. Then the subset $\{W\}$ of $\mathcal{P}$ induced by the sequence $W$ is defined to be

$$
\{W\}=\left\{p_{1}, \ldots, p_{N}\right\} \text { in } \mathcal{P} \text {. }
$$

For example, if $W=(2,3,2,3,7)$ in $\mathcal{P}^{5}$, then $\{W\}=\{2,3,7\}$ in $\mathcal{P}$.

Similarly, for a finite sequence $J=\left(j_{1}, \ldots, j_{N}\right)$ in $\mathbb{Z}$, one can determine the corresponding subset $\{J\}=\left\{j_{1}, \ldots, j_{N}\right\}$ of $\mathbb{Z}$, for all $N \in \mathbb{N}$.

Let $\eta=\left(n_{1}, \ldots, n_{N}\right)$ be a finite sequence in $\mathbb{N}$, and let

$$
\{\eta\}=\left\{n_{1}, \ldots, n_{N}\right\} \text { in } \mathbb{N}
$$

for $N \in \mathbb{N}$.

If we fix

$$
|W|=|J|=|\eta|=N \text {, for some } N \in \mathbb{N},
$$

and

$$
W=\left(p_{1}, \ldots, p_{N}\right), J=\left(j_{1}, \ldots, j_{N}\right), \eta=\left(n_{1}, \ldots, n_{N}\right),
$$

where |.| means the size or the length of finite sequences, then one can define the corresponding operators $X_{W}^{J: \eta}$ and $M_{W}^{J: \eta}$ of $\mathfrak{L S}$ by

$$
X_{W}^{J: \eta}=\prod_{l=1}^{N} Q_{p_{l}, j_{l}}^{n_{l}}, \text { and } M_{W}^{J: \eta}=\prod_{l=1}^{N} \Theta_{p_{l}, j_{l}}^{n_{l}}
$$

in $\mathfrak{L} \mathfrak{S}$, generated by $Q$, respectively, by $\Theta$ of (11.1).
The construction (11.2) shows that if $N>1$ in $\mathbb{N}$, then $X_{W}^{J: \eta}$ and $M_{W}^{J: \eta}$ are the operators in the sense of (10.2), respectively (9.1). While, if $N=1$, then

$$
X_{W}^{J: \eta}=Q_{p_{l}, j_{l}}^{n_{l}} \text {, and } M_{W}^{J: \eta}=\Theta_{p_{l}, j_{l}}^{n_{l}},
$$

in the free block $\mathfrak{L S}_{p_{1}}\left(j_{1}\right) \subset \mathfrak{L} \mathfrak{S}$, which is the very minimal free summand of $\mathfrak{L} \mathfrak{S}$ containing them.

So, the operators formed by (11.2) are not only the operators in Sections 9 and 10, but also our weighted-semicircular, and semicircular elements in Sections 7 and 8 in $\mathfrak{L} \mathfrak{S}$.

Definition 11.1. Let $X_{W}^{J: \eta}$ and $M_{W}^{J: \eta}$ be in the sense of (11.2) in $\mathfrak{L S}$, for a fixed $N \in \mathbb{N}$. If $X_{W}^{J: \eta}$ (or, $M_{W}^{J: \eta}$ ) is a free reduced word in $\mathfrak{L} \mathfrak{S}$, or equivalently, if either $W$ or $J$ is alternating, then the operator $X_{W}^{J: \eta}$ (or, $M_{W}^{J: \eta}$ ) is called a weighted-semicircular(resp., a semicircular) reduced word of $\mathfrak{L} \mathfrak{S}$ generated by $\mathcal{Q}$ (resp., by $\Theta$ )).

Moreover, if " $N>1$," and if either $p_{N} \neq p_{1}$ in $\mathcal{P}$, or $j_{N} \neq j_{1}$ in $\mathbb{Z}$, then the corresponding weighted-semicircular reduced word $X_{W}^{J: \eta}$ (resp., the corresponding semicircular reduced word $M_{W}^{J: \eta}$ ) is said to be a power-w(eighted)s(emicircular) reduced word (resp., a power-s(emicircular) reduced word) of $\mathfrak{L} \mathfrak{S}$ generated by $\mathcal{Q}$ (resp., by $\Theta$ ). Remark that power-w-s reduced words and power-s reduced words are defined only if the free reduced words have their length $N>1$.

If $X_{W}^{J: \eta}$ is a power-w-s reduced word of $\mathfrak{L} \mathfrak{S}$, then $X_{W}^{J: \eta}$ satisfies

$$
\begin{aligned}
& \tau^{0}\left(\left(X_{W}^{J: \eta}\right)^{(k)}\right)=\prod_{l=1}^{N} \omega_{n_{l}}\left(p_{l}^{n_{l}\left(j_{l}+1\right)} c_{\frac{n_{l}}{2}}\right)^{k} \\
& =\tau^{0}\left(\left(\left(X_{W}^{J: \eta}\right)^{(*)}\right)^{(k)}\right)
\end{aligned}
$$

in $\mathfrak{L} \mathcal{S}$, by (10.3) and (10.5), and if $M_{W}^{J: \eta}$ is a power-s reduced word of $\mathfrak{L S}$, then (11.4)

$$
\begin{aligned}
& \tau^{0}\left(\left(M_{W}^{J: \eta}\right)^{(k)}\right)=\prod_{l=1}^{N} \omega_{n_{l}}\left(\frac{c_{n_{l}}}{2}\right)^{k} \\
& =\tau^{0}\left(\left(\left(M_{W}^{J: \eta}\right)^{(*)}\right)^{(k)}\right),
\end{aligned}
$$

by (9.2) and (9.3), for all $k \in \mathbb{N}$.

Definition 11.2. Let $X_{W}^{J: \eta}$ and $M_{W}^{J: \eta}$ be a weightedsemicircular reduced word (not necessarily power-w-s), 
and a semicircular reduced word (not necessarily power-s) in $\mathfrak{L} \mathfrak{S}$, respectively. Define a sequence $r_{W}^{J: \eta}[\mathcal{Q}: \Theta]$ by

$$
r_{W}^{J: \eta}[\mathcal{Q}: \Theta]=\left(\frac{\operatorname{def}}{\tau^{0}\left(\left(M_{W}^{J: \eta}\right)^{(k)}\right)}\right)_{k=1}\left(X_{W}^{J: \eta}\right)^{(k)},
$$

with axiomatization:

$$
\frac{0}{0}=0 \text { in } \mathbb{C}
$$

We call this sequence $r_{W}^{J: \eta}[\mathcal{Q}: \Theta]$ of (11.5), the weight-ratio of $X_{W}^{J: \eta}$ and $M_{W}^{J: \eta}$ in $\mathfrak{L} \mathfrak{S}$.

Observe that, by the definition (11.5) (with axiomatization: $\frac{0}{0}=0$ ), if $X_{W}^{J: \eta}$ and $M_{W}^{J: \eta}$ are a power-w-s reduced word, respectively, a power-s reduced word in $\mathfrak{L S}$, then

$$
\begin{aligned}
& r_{W}^{J: \eta}[\mathcal{Q}: \Theta]=\left(\frac{\tau^{0}\left(\left(X_{W}^{J: \eta}\right)^{(k)}\right)}{\tau^{0}\left(\left(M_{W}^{J: \eta}\right)^{(k)}\right)}\right)_{k=1}^{\infty} \\
& =\left(\frac{\prod_{l=1}^{N} \omega_{n_{l}}\left(p_{l}^{n_{l}\left(j_{l}+1\right)} c_{\frac{n_{l}}{2}}\right)^{k}}{\prod_{l=1}^{N} \omega_{n_{l}}\left(c_{n_{l}}\right)^{k}}\right)_{k=1}^{\infty}
\end{aligned}
$$

by (11.3) and (11.4)

$$
=\left(\frac{\omega_{k \eta}\left(\prod_{l=1}^{N} p_{l}^{n_{l}\left(j_{l}+1\right)} c_{\frac{n_{l}}{2}}\right)^{k}}{\omega_{k \eta}\left(\prod_{l=1}^{N} c_{n_{l}}\right)^{k}}\right)_{k=1}^{\infty}
$$

where

$$
\omega_{k \eta} \stackrel{\text { def }}{=} \begin{cases}0 & \text { if } \exists n_{l} \in\{\eta\} \text { s.t., } n_{l} \text { is odd } \\ 1 & \text { if } n_{l} \text { are even, } \forall n_{l} \in\{\eta\},\end{cases}
$$

for all $k \in \mathbb{N}$, and then

$$
=\left(\omega_{k \eta}\left(\prod_{l=1}^{N} p_{l}^{n_{l}\left(j_{l}+1\right)}\right)^{k}\right)_{k=1}^{\infty},
$$

by (11.6), i.e.,

$$
r_{W}^{J: \eta}[\mathcal{Q}: \Theta]=\left(\omega_{k \eta}\left(\prod_{l=1}^{N} p_{l}^{k n_{l}\left(j_{l}+1\right)}\right)\right)_{k=1}^{\infty},
$$

where $\left(\omega_{k \eta}\right)_{k=1}^{\infty}$ is in the sense of (11.6).

Proposition 11.1. Let $r_{W}^{J: \eta}[\mathcal{Q}: \Theta]$ be the weight-ratio (11.5) of a power-w-s reduced word $X_{W}^{J: \eta}$ and a power-s reduced word $M_{W}^{J: \eta}$ in the sense of (11.2). Then

$$
r_{W}^{J: \eta}[\mathcal{Q}: \Theta]=\left(\omega_{k \eta}\left(\prod_{l=1}^{N} p_{l}^{n_{l}\left(j_{l}+1\right)}\right)^{k}\right)_{k=1}^{\infty},
$$

where $\left(\omega_{k \eta}\right)_{k=1}^{\infty}$ is in the sense of (11.6).

Proof. The proof of (11.8) is done by (11.7), under our axiomatization: $\frac{0}{0}=0$.

Note that if $X_{W}^{J: \eta}$ is a weighted-semicircular reduced word (which is not necessarily power-w-s), and if $M_{W}^{J: \eta}$ is a semicircular reduced word (which is not necessarily power-s) in $\mathfrak{L S}$, then the above relation (11.8) for $r_{W}^{J: \eta}[\mathcal{Q}: \Theta]$ does not hold in general.

We will denote the $k$-th entry of the weight-ratio $r_{W}^{J: \eta}[\mathcal{Q}: \Theta]$ of $X_{W}^{J: \eta}$ and $M_{W}^{J: \eta}$ by

$$
r_{W}^{J: \eta}[\mathcal{Q}: \Theta] \text {, for all } k \in \mathbb{N} .
$$

So, if $X_{W}^{J: \eta}$ and $M_{W}^{J: \eta}$ are a power-w-s reduced word, respectively, a power-s reduced word in $\mathfrak{L} \mathfrak{S}$, then

$$
r_{W}^{J: \eta}[k ; \mathcal{Q}: \Theta]=\omega_{k \eta}\left(\prod_{l=1}^{N} p_{l}^{n_{l}\left(j_{l}+1\right)}\right)^{k},
$$

by (11.7) and (11.9), for all $k \in \mathbb{N}$.

By definition, the $k$-th entry (11.9) of the weight-ratio $r_{W}^{J: \eta}[\mathcal{Q}: \Theta]$ shows how the $k$-th free moment of $X_{W}^{J: \eta}$ and that of $M_{W}^{J: \eta}$ are different free-distributionally. In other words, the sequence $r_{W}^{J: \eta}[\mathcal{Q}: \Theta]$ indicates how the free distribution of $X_{W}^{J: \eta}$ and that of $M_{W}^{J: \eta}$ can be distinguished.

Suppose one takes $\eta=\left(n_{1}, \ldots, n_{N}\right)$ be a finite sequence of "even" numbers, i.e., $n_{l} \in 2 \mathbb{N}$, for all $l=1, \ldots N$. In such a case, one may / can say the $N$-tuple $\eta$ is even, for convenience.

If $X_{W}^{J: \eta}$ is a power-w-s reduced word, and hence, $M_{W}^{J: \eta}$ is a power-s reduced word in $\mathfrak{L} \mathfrak{S}$, and if $\eta$ is even, then

$$
r_{W}^{J: \eta}[\mathcal{Q}: \Theta]=\left(\left(\prod_{l=1}^{N} p_{l}^{n_{l}\left(j_{l}+1\right)}\right)^{k}\right)_{k=1}^{\infty},
$$




$$
\omega_{k \eta}=1 \text {, for all } k \in \mathbb{N} \text {. }
$$

Corollary 11.2. Let $X_{W}^{J: \eta}$ and $M_{W}^{J: \eta}$ be a power-w-s reduced word, respectively, a power-s reduced word in $\mathfrak{L S}$, and let $r_{W}^{J: \eta}[\mathcal{Q}: \Theta]$ be the weight-ratio of $X_{W}^{J: \eta}$ and $M_{W}^{J: \eta}$. If $\eta$ is even, then

$$
r_{W}^{J: \eta}[\mathcal{Q}: \Theta]=\left(\prod_{l=1}^{N}\left(p_{l}^{n_{l}\left(j_{l}+1\right)}\right)^{k}\right)_{k=1}^{\infty} .
$$

Let $r_{W}^{J: \eta}[\mathcal{Q}: \Theta]$ be the weight-ratio (11.5). By understanding it as a R-sequence, one can consider convergence, or divergence of the sequence. Note that every entry $r_{W}^{J: \eta}[k ; \mathcal{Q}: \Theta]$ of $r_{W}^{J: \eta}[\mathcal{Q}: \Theta]$ is a nonnegative real number.

If $r_{W}^{J: \eta}[\mathcal{Q}: \Theta]$ is the weight-ratio of a power-w-s reduced word $X_{W}^{J: \eta}$ and a power-s reduced word $M_{W}^{J: \eta}$, and if we denote the first entry $r_{W}^{J: \eta}[1 ; \mathcal{Q}: \Theta]$,

$$
\prod_{l=1}^{N} \omega_{k \eta} p_{l}^{n_{l}\left(j_{l}+1\right)} \text { by } r_{0} \text { in } \mathbb{R}
$$

then one obtains that

$$
r_{W}^{J: \eta}[\mathcal{Q}: \Theta]=\left(r_{0}^{k}\right)_{k=1}^{\infty},
$$

by (11.8). Thus, one can have the following cases; (i) if $r_{0}=0$, then

$$
\lim _{k \rightarrow \infty} r_{W}^{J: \eta}[k ; \mathcal{Q}: \Theta]=\lim _{k \rightarrow \infty} r_{0}^{k}=\lim _{k \rightarrow \infty} 0=0 ;
$$

(ii) if $r_{0}=1$, then

$$
\lim _{k \rightarrow \infty} r_{W}^{J: \eta}[k ; \mathcal{Q}: \Theta]=\lim _{k \rightarrow \infty} r_{0}^{k}=\lim _{k \rightarrow \infty} 1=1 ;
$$

(iii) if $r_{0}>1$, then

$$
\lim _{k \rightarrow \infty} r_{W}^{J: \eta}[k ; \mathcal{Q}: \Theta]=\lim _{k \rightarrow \infty} r_{0}^{k}=\infty ;
$$

and (iv) if $0<r_{0}<1$, then

$$
\lim _{k \rightarrow \infty} r_{W}^{J: \eta}[k ; \mathcal{Q}: \Theta]=\lim _{k \rightarrow \infty} r_{0}^{k}=0
$$

Proposition 11.3. Let $N>1$ in $\mathbb{N}$, and let $X_{W}^{J: \eta}$ and $M_{W}^{J: \eta}$ be a power-w-s reduced word, and a power-s reduced word in $\mathfrak{L S}$, respectively. Let $r_{W}^{J: \eta}[\mathcal{Q}: \Theta]$ be the weightratio of $X_{W}^{J: \eta}$ and $M_{W}^{J: \eta}$, and let

$$
r_{0}=\prod_{l=1}^{N} \omega_{n_{l}} p_{l}^{n_{l}\left(j_{l}+1\right)} \text { in } \mathbb{R} \text {. }
$$

(11.12) If $r_{0}=1$, then $r_{W}^{J: \eta}[\mathcal{Q}: \Theta]$ converges to 1 .

(11.13) If $r_{0}>1$, then $r_{W}^{J: \eta}[\mathcal{Q}: \Theta]$ diverges to $\infty$.
(11.14) If $0 \leq r_{0}<1$, then $r_{W}^{J: \eta}[\mathcal{Q}: \Theta]$ converges to 0 .

Proof. Under hypothesis, let $r_{0}=\prod_{l=1}^{N} \omega_{n_{l}} p_{l}^{n_{l}\left(j_{l}+1\right)}$ in $\mathbb{R}$. Then, by (11.8) and (11.11), one has that

$$
r_{W}^{J: \eta}[\mathcal{Q}: \Theta]=\left(r_{0}^{k}\right)_{k=1}^{\infty} .
$$

So, by the discussions (i), (ii), (iii) and (iv) in the very above paragraph, we obtain the convergence conditions (11.12), (11.13) and (11.14) of our weight-ratio $r_{W}^{J: \eta}[\mathcal{Q}: \Theta]$.

Perhaps, by the convergence conditions (11.12), (11.13) and (11.14), one may /can say our power-w-s reduced word $X_{W}^{J: \eta}$ is

$$
\left(\lim _{k \rightarrow \infty} r_{W}^{J: \eta}[\mathcal{Q}: \Theta]\right)-\text { close }
$$

to a power-s reduced word $M_{W}^{J: \eta}$ in the radial-Adelic probability space $\mathfrak{L} \mathfrak{S}$. In other words, we may / can have three characterizations for close-ness determined by (11.12), (11.13) and (11.14). For instance, by (11.14), one may / can conclude that: if the nonnegative real quantity

$$
r_{0}=\prod_{l=1}^{N} \omega_{n_{l}} p_{l}^{n_{l}\left(j_{l}+1\right)}
$$

satisfies

$$
0 \leq r_{0}<1 \text { in } \mathbb{R}
$$

then the free distribution of $X_{W}^{J: \eta}$ is close to the free distribution of $M_{W}^{J: \eta}$, and the close-ness may / can be measured by the limit 0 of the weight-ratio $r_{W}^{J: \eta}[\mathcal{Q}: \Theta]$ (which means the free distribution of $X_{W}^{J: \eta}$ (determined by weighted-semicircularity) is "very" much close, or 0 -much close to that of $M_{W}^{J: \eta}$ (determined by semicircularity)). However, such conclusions, or estimations are too rough. So, we provide a following new way to determine the close-ness, motivated by (11.11).

Definition 11.3. Let $r_{W}^{J: \eta}[\mathcal{Q}: \Theta]$ be the weight-ratio of a weighted-semicircular reduced word $X_{W}^{J: \eta}$ and a semicircular reduced word $M_{W}^{J: \eta}$ (which are not necessarily power-w-s, respectively, power-s) of $\mathfrak{L} \mathfrak{S}$. As a R-sequence, let

$$
r_{W}^{J: \eta}[\mathcal{Q}: \Theta]=\left(r_{n}\right)_{n=1}^{\infty}
$$

and suppose

$$
\beta_{0}=\lim _{k \rightarrow \infty}\left(\max \left\{r_{1}, \ldots, r_{k}\right\}\right), \text { in } \mathbb{R} \cup\{\infty\} .
$$

If $\beta_{0}<\infty$, then $X_{W}^{J: \eta}$ is said to be close to $M_{W}^{J: \eta}$ with its ratio $\beta_{0}$ (in short, $X_{W}^{J: \eta}$ is $\beta_{0}$-close to $M_{W}^{J: \eta}$ ) in $\mathfrak{L} \mathfrak{S}$. Otherwise, we say $X_{W}^{J: \eta}$ is not close to $M_{W}^{J: \eta}$ in $\mathfrak{L} \mathfrak{S}$. 
By definition, a weighted-semicircular reduced word $X_{W}^{J: \eta}$ is $\beta_{0}$-close to a semicircular reduced word $M_{W}^{J: \eta}$ in $\mathfrak{L S}$, if and only if the free distribution of $X_{W}^{J: \eta}$ is close to that of $M_{W}^{J: \eta}$, and the close-ness can be measured by the nonnegative quantity

$$
\beta_{0}=\lim _{k \rightarrow \infty}\left(\max \left\{r_{W}^{J: \eta}[1 ; \mathcal{Q}: \Theta], \ldots, r_{W}^{J: \eta}[k ; \mathcal{Q}: \Theta]\right\}\right),
$$

in $\mathbb{R} \cup\{\infty\}$.

Let $W=(p), \quad J=(j)$, and $\eta=(n)$, and let

$$
X \stackrel{\text { denote }}{=} X_{W}^{J: \eta}=Q_{p, j}^{n}=Q_{p, j}^{(n)},
$$

and

$$
M \stackrel{\text { denote }}{=} M_{W}^{J: \eta}=\Theta_{p, j}^{n}=\Theta_{p, j}^{(n)},
$$

be the corresponding weighted-semicircular reduced word, respectively, semicircular reduced word in $\mathfrak{L} \mathfrak{S}$. If two operators $X$ and $M$ are in the sense of (11.15), then

$$
X^{k}=Q_{p, j}^{k n}=X^{(k)} \text {, and } M^{k}=\Theta_{p, j}^{k n}=M^{(k)},(11
$$

in the free block $\mathfrak{L S}_{p}(j)$ in $\mathfrak{L S}$, for all $k \in \mathbb{N}$ (See Section 8.1).

These operators $X$ and $M$ of (11.15) have their free distributions,

$$
\left\{\tau^{0}\left(X^{(k)}\right)=\tau^{0}\left(X^{k}\right)=\omega_{k n} p^{k n(j+1)} c_{k n}\right\}_{k=1}^{\infty},
$$

respectively,

$$
\left\{\tau^{0}\left(M^{(k)}\right)=\tau^{0}\left(M^{k}\right)=\omega_{k n} c_{\frac{k n}{2}}\right\}_{k=1}^{\infty},
$$

by (11.15)'.

By the free distributions (11.16), the operators $X$ and $M$ of (11.15) have their weight-ratio,

$$
r_{W}^{J: \eta}[\mathcal{Q}: \Theta]=\left(\omega_{k n} p^{k n(j+1)}\right)_{k=1}^{\infty},
$$

under axiomatization: $\frac{0}{0}=0$.

Thus one can obtain the following close-ness of $X$ to $M$.

Theorem 11.4. Let $X=Q_{p, j}^{n}$ and $M=\Theta_{p, j}^{n}$ in the radial-Adelic probability space $\mathfrak{L} \mathfrak{S}$, where $Q_{p, j}$ is a $p^{2(j+1)}$-semicircular element in $\mathcal{Q}$, and $\Theta_{p, j}$ is a semicircular element in $\Theta$, for $j \in \mathbb{Z}$ and $p \in \mathcal{P}$, for $a$ fixed $n \in \mathbb{N}$.

(11.18) If $j=-1$ in $\mathbb{Z}$, then $X$ is 1 -close to $M$ in $\mathfrak{L} \mathfrak{S}$.
(11.19) If $j<-1$ in $\mathbb{Z}$, then there exists $k_{0} \in \mathbb{N}$, such that (i) $k_{0}$ is the minimal quantity satisfying $\omega_{k_{0} n}=1$, and (ii) $X$ is $p^{k_{0} n(j+1)}$-close to $M$ in $\mathfrak{L} \mathfrak{S}$.

(11.20) If $j>-1$ in $\mathbb{Z}$, then $X$ is not close to $M$ in $\mathfrak{L} \mathfrak{S}$. Proof. Suppose $n \in \mathbb{N}$ is fixed, and let $X$ and $M$ be given as above in $\mathfrak{L} \mathfrak{S}$. Then the weight-ratio $R$ of $X$ and $M$ satisfies

$$
R=r_{(p)}^{(j):(n)}[\mathcal{Q}: \Theta]=\left(\omega_{k n} p^{k n(j+1)}\right)_{k=1}^{\infty}
$$

by (11.17).

Assume first that $j=-1$ in $\mathbb{Z}$. Then the above weight-ratio $R$ of (11.21) satisfies that

$$
R=\left(\omega_{k n} \cdot 1\right)_{k=1}^{\infty}=\left(\omega_{k n}\right)_{k=1}^{\infty} .
$$

So, one obtains that there exists $k_{0} \in \mathbb{N}$, such that (i) it is the minimal quantity satisfying $\omega_{k_{0} n}=1$, and (ii)

$$
\begin{aligned}
& \lim _{k \rightarrow \infty}\left(\max \left\{\omega_{n}, \omega_{2 n}, \ldots, \omega_{k n}\right\}\right) \\
& =\lim _{k \rightarrow \infty}\left(\max \left\{0, \ldots, 0, \omega_{k_{0} n}=1, \omega_{\left(k_{0}+1\right) n}, \ldots, \omega_{k}\right\}\right) \\
& =\lim _{k \rightarrow \infty} 1=1,
\end{aligned}
$$

because $\omega_{k} \in\{0,1\}$, for all $k \in \mathbb{N}$. The existence of such quantity $k_{0}$ is guaranteed, because for any natural number $k \in \mathbb{N}$, there always exist $k^{\prime} \in \mathbb{N}$, such that $k^{\prime} k$ is even in $\mathbb{N}$.

It shows that if $j=-1$ in $\mathrm{Z}$; then $\mathbb{Z}$, is 1 -close to $M$ in $\mathfrak{L S}$, and hence, the statement (11.18) holds.

Now, suppose $j<-1$ in $\mathbb{Z}$. Then the weight-ratio $R$ of (11.21) satisfies that

$$
R=\left(\omega_{k n} p^{k n(j+1)}\right)_{k=1}^{\infty}=\left(\frac{\omega_{k n}}{p^{k n|j+1|}}\right)_{k=1}^{\infty},
$$

because $j+1<0$ in $\mathbb{R}$, where $|j+1|$ means the absolute value of $j+1$ in $\mathbb{R}$.

So, by the similar manner with the proof of (11.18), there exists $k_{0} \in \mathbb{N}$, such that (i) $k_{0}$ is the minimal quantity satisfying $\omega_{k_{0} n}=1$, and (ii)

$$
\begin{aligned}
& \lim _{k \rightarrow \infty}\left(\max \left\{0, \ldots, 0, \frac{\omega_{k_{0} n}}{p^{k_{0} n|j+1|}}, \frac{\omega_{\left(k_{0}+1\right) n}}{p^{\left(k_{0}+1\right) n|j+1|}}, \ldots, \frac{\omega_{k n}}{p^{k n|j+1|}}\right\}\right) \\
& =\lim _{k \rightarrow \infty}\left(\frac{1}{p^{k_{0} n|j+1|}}\right)=p^{k_{0} n(j+1)},
\end{aligned}
$$

since the weight-ratio $R$ forms a monotonically decreasing sequence in $\mathbb{R}$.

It shows that, if $j<-1$ in $\mathbb{Z}$, then there exists a unique $k_{0} \in \mathbb{N}$, such that $X$ is $p^{k_{0}^{n}(j+1)}$-close to $M$ in $\mathfrak{L} \mathfrak{S}$, where $k_{0}$ is the minimal quantity satisfying $\omega_{k_{0} n}=1$. Therefore, the statement (11.19) holds true. 
Finally, assume that $j>-1$ in $\mathbb{Z}$. Then the weight-ratio

$$
R=\left(\omega_{k n} p^{k n(j+1)}\right)_{k=1}^{\infty}=\left(t_{k}\right)_{k=1}^{\infty}
$$

of (11.21) contains a sub-sequence $R_{+}$,

$$
R_{+}=\left(p^{k_{0} n(j+1)}, p^{2 k_{0} n(j+1)}, \ldots\right)^{\text {denote }}=\left(s_{k}\right)_{k=1}^{\infty}
$$

which is a monotonically "strictly" increasing sequence in $\mathbb{R}$, where $k_{0}$ is the minimal quantity in $\mathbb{N}$, satisfying $\omega_{k_{0} n}=1$.

Since the weight-ratio $R$ is a monotonically increasing sequence, one can get that

$$
\begin{aligned}
\infty & =\lim _{k \rightarrow \infty} s_{k}=\lim _{k \rightarrow \infty}\left(\max \left\{s_{1}, \ldots, s_{k}\right\}\right) \\
& \leq \lim _{k \rightarrow \infty}\left(\max \left\{t_{1}, \ldots, t_{k}\right\}\right),
\end{aligned}
$$

by the monotonically-strictly-increasing-ness of the sub-sequence $R_{+}$of $R$, which implies that

$$
\lim _{k \rightarrow \infty}\left(\max \left\{t_{1}, \ldots, t_{k}\right\}\right)=\infty .
$$

So, $X$ is not close to $M$ in $\mathfrak{L} \mathfrak{S}$. Thus, the statement (11.20) holds.

The above theorem, expressed by the close-ness conditions (11.18), (11.19) and (11.20), characterizes how much our weighted-semicircular element $Q_{p, j}$ s close to a semicircular element $\Theta_{p, j}$ in $\mathfrak{L} \mathfrak{S}$, for $j \in \mathbb{Z}, p \in \mathcal{P}$.

Corollary 11.5. Let $p \in \mathcal{P}$, and $j \in \mathbb{Z}$, and let $Q_{p, j}$ be the $p^{2(j+1)}$-semicircular element, and $\Theta_{p, j}$, the semicircular element in $\mathfrak{L} \mathfrak{S}$.

(11.22) If $j=-1$ in $\mathbb{Z}$, then $Q_{p, j}$ is 1 -close to $\Theta_{p, j}$ in $\mathfrak{L} \mathfrak{S}$.

(11.23) If $j<-1$ in $\mathbb{Z}$, then $Q_{p, j}$ is $p^{2(j+1)}$-close to $\Theta_{p, j}$ in $\mathfrak{L} \mathfrak{S}$.

(11.24) If $j>-1$ in $\mathbb{Z}$, then $Q_{p, j}$ is not close to $\Theta_{p, j}$ in $\mathfrak{L} \mathfrak{S}$.

Proof. The proofs of (11.22), (11.23) and (11.24) are done by those of (11.18), (11.19) and (11.20), respectively, by putting $n=1$. Remark that, in this case, the weight-ratio satisfies that

$$
r_{(p)}^{(j):(1)}[\mathcal{Q}: \Theta]=\left(0, p^{2(j+1)}, 0, p^{4(j+1)}, 0, p^{6(j+1)}, \ldots\right) .
$$

The above corollary, expressed by (11.22), (11.23) and (11.24), illustrates the close-ness of our weighted-semicircular elements $Q_{p, j}$ and the corresponding semicircular elements $\Theta_{p, j}$.

More generally, one can obtain the following corollary in terms of identically-free-distributedness.

Corollary 11.6. Let $X=X_{(p)}^{(j):(n)}$ be our weightedsemicircular reduced word (11.2) in $\mathfrak{L} \mathfrak{S}$, for $j \in \mathbb{Z}$, $n \in \mathbb{N}, \quad p \in \mathcal{P}$, and let $s$ be an arbitrary semicircular element in a topological *-probability space $(A, \varphi)$. Define a sequence $r\left(X, s^{n}\right)$ of $X$ and $s^{n}$ by

$$
\begin{aligned}
& r\left(X: s^{n}\right)=\left(\frac{\tau^{0}\left(X^{(k)}\right)}{\varphi\left(s^{k}\right)}\right)_{k=1}^{\infty} \\
& =\left(\frac{\tau^{0}\left(X^{k}\right)}{\varphi\left(s^{k}\right)}\right)_{k=1}^{\infty}=\left(t_{k}\right)_{k=1}^{\infty},
\end{aligned}
$$

under axiomatization: $\frac{0}{0}=0$.

Also, define a $\mathbb{R}$-sequence

$$
\left(m_{k}\right)_{k=1}^{\infty}=\left(\max \left\{t_{1}, \ldots, t_{k}\right\}\right)_{k=1}^{\infty},
$$

where $t_{l}$ 's are in the sense of (11.25). Then

$$
\lim _{k \rightarrow \infty} m_{k}= \begin{cases}1 & \text { if } j=-1 \text { in } \mathbb{Z} \\ p^{k_{0} n(j+1)} & \text { if } j<-1 \text { in } \mathbb{Z}, \\ \infty & \text { if } j>-1 \text { in } \mathbb{Z}\end{cases}
$$

where $k_{0}$ is the minimal quantity satisfying $\omega_{k_{0} n}=1$ in $\mathbb{N}$. Therefore, up to (11.25), we may / can say that $X$ is $\left(\lim _{k \rightarrow \infty} m_{k}\right)$-close to $s^{n}$, for the fixed $n \in \mathbb{N}$, where $\lim _{k \rightarrow \infty} m_{k}$ are in the sense of (11.26).

Proof. If $r\left(X: s^{n}\right)=\left(t_{k}\right)_{k=1}^{\infty}$ is a $\mathbb{R}$-sequence (11.25), where $X$ is the weighted-semicircular reduced word $X_{(p)}^{(j):(n)}$, and $s^{n}$ is the $n$-th power of an arbitrary semicircular element $s$ in a topological *-probability space $(A, \varphi)$. And let

$$
\left(m_{k}\right)_{k=1}^{\infty}=\left(\max \left\{t_{1}, \ldots, t_{k}\right\}\right)_{k=1}^{\infty} .
$$

Since $s^{n}$ and our free semicircular reduced word $M=M_{(p)}^{(j):(n)}=\Theta_{p, j}^{n}$ are identically free-distributed, the sequence $r\left(X: s^{n}\right)$ of (11.25) is identical to our weightratio $r_{(p)}^{(j):(n)}[\mathcal{Q}: \Theta]$, as $\mathbb{R}$-sequences.

Therefore, one obtains the formula (11.26), by (11.18), (11.19) and (11.20).

The above corollary shows how our weightedsemicircularity (on $\mathfrak{L} \mathfrak{S}$ ) differs (or, is close) from (resp., to) the semicircular law, and the difference (or the closeness) of them is measured by (11.26). Thus, by abusing notation, for example, one can/ may say that: a weightedsemicircular reduced word $X_{(p)}^{(j):(n)}$ of $\mathfrak{L} \mathfrak{S}$ is $p^{k_{0} n(j+1)}$ - 
close to the $n$-th power $s^{n}$ of any arbitrary semicircular element $s$, whenever $j \leq-1$ in $\mathbb{Z}$, for all $n \in \mathbb{N}$.

The following proposition precisely determine the minimal quantity $k_{0}$ obtained in our close-ness conditions (11.19) and (11.26).

Proposition 11.7. Let $X=X_{(p)}^{(j):(n)}=Q_{p, j}^{n}$ be our $p^{2(j+1)}$-semicircular reduced word in the radial-Adelic probability space $\mathfrak{L} \mathfrak{S}$, and assume that $j<-1$ in $\mathbb{Z}$, and hence, $X$ is $p^{k_{0} n(j+1)}$-close to any semicircular element $s$ in a topological *-probability space $(A, \varphi)$, where $k_{0}$ is the minimal quantity satisfying $\omega_{k_{0} n}=1$. Then

$$
k_{0}=\left\{\begin{array}{l}
1 \text { if } n \text { is even } \\
2 \text { if } n \text { is odd, }
\end{array}\right.
$$

for all $n \in \mathbb{N}$. It means that, if $j<-1$ in $\mathbb{Z}$, then $X$ is either $p^{n(j+1)}$-close, or $p^{2 n(j+1)}$-close to $s$.

Proof. Suppose $n$ is even. Then $\omega_{k n}=1$, for all $k \in \mathbb{N}$, and hence, 1 is the minimal quantity making $\omega_{1 \cdot n}=1$. Assume now that $n$ is odd. Then $\omega_{k n}=1$, if and only if $k n$ is even in $\mathbb{N}$. So, the smallest even quantity $k n$ is $2 n$ in $\mathbb{N}$. Therefore, 2 is the minimal quantity making $\omega_{2 \cdot n}=1$. So, the above equality (11.27) holds.

The equality (11.27) completely characterizes the minimal quantity $k_{0}$ making $\omega_{k_{0} n}=1$, for all $n \in \mathbb{N}$.

Corollary 11.8. Let $X$ be a weighted-semicircular reduced word $X_{(p)}^{(j):(n)}=Q_{p, j}^{n}$ in $\mathfrak{L} \mathfrak{S}$, for $j \in \mathbb{Z}, \quad p \in \mathcal{P}$, for $a$ fixed $n \in \mathbb{N}$. Assume that $j<-1$ in $\mathbb{Z}$.

(11.28) If $n \in 2 \mathbb{N}$, then $X$ is $p^{n(j+1)}$-close to $\Theta_{p, j}^{n}$ in $\mathfrak{L S}$.

(11.29) If $n \in 2 \mathbb{N}-1$, then $X$ is $p^{2 n(j+1)}$-close to $\Theta_{p, j}^{n}$, in $\mathfrak{L} \mathfrak{S}$,

where $\Theta_{p, j}$ is the semicircular element $\frac{1}{p^{j+1}} Q_{p, j}$ in $\mathfrak{L S}$.

Proof. The statements (11.28) and (11.29) are the direct consequences of (11.19) and (11.27).

Now, assume that $\mathrm{N}>1$ in $\mathbb{N}$; and let

$$
X_{N} \stackrel{\text { denote }}{=} X_{W}^{J: \eta}=\prod_{l=1}^{N} Q_{p_{l}, j_{l}}^{n_{l}}
$$

and

$$
M_{N} \stackrel{\text { denote }}{=} M_{W}^{J: \eta}=\prod_{l=1}^{N} \Theta_{p_{l}, j_{l}}^{n_{l}}
$$

be power-w-s, respectively, power-s reduced words in $\mathfrak{L} \mathfrak{S}$. Then, the weight-ratio satisfies

$$
R_{N} \stackrel{\text { denote }}{=} r_{W}^{J: \eta}[\mathcal{Q}: \Theta]=\left(\left(\prod_{l=1}^{N} \omega_{n_{l}} p_{l}^{n_{l}\left(j_{l}+1\right)}\right)^{k}\right)_{k=1}^{\infty},
$$

by (11.8). So, if we write

$$
r_{0}=\prod_{l=1}^{N} \omega_{n_{l}} p^{n_{l}\left(j_{l}+1\right)} \text { in } \mathbb{R}
$$

then

$$
R_{N}=\left(r_{0}^{k}\right)_{k=1}^{\infty}
$$

as a $\mathbb{R}$-sequence.

Lemma 11.9. Let $X_{N}$ and $M_{N}$ be a power-w-s, respectively, a power-s reduced words (11.30) in $\mathfrak{L S}$, and let $R_{N}=r_{W}^{J: \eta}[\mathcal{Q}: \Theta]$ be the weight-ratio of $X_{N}$ and $M_{N}$. If there exists at least one $n_{l_{0}} \in\{\eta\}$, such that $n_{l_{0}}$ is odd in $\mathbb{N}$, then $R_{N}$ is the zero sequence, i.e.,

$$
R_{N}=(0,0,0, \ldots)
$$

Proof. Let $X_{N}=X_{W}^{J: \eta}$ and $M_{N}=M_{W}^{J: \eta}$ be given as above in $\mathfrak{L} \mathfrak{S}$, with $N=|\eta|>1$ in $\mathbb{N}$. Assume now that there exists at least one $n_{l_{0}} \in\{\eta\}$, such that $n_{l_{0}} \in 2 \mathbb{N}-1$. Then the quantity $r_{0}$ of (11.31) is identical to 0 . since $\omega_{n_{l_{0}}}=0$. So, the corresponding weight-ratio $R_{N}$ satisfies that

$$
R_{N}=\left(r_{0}^{k}\right)_{k=1}^{\infty}=\left(0^{k}\right)_{k=1}^{\infty}=(0,0,0, \ldots)
$$

By the above lemma, one can obtain the following close-ness condition.

Theorem 11.10. Let $X_{N}$ and $M_{N}$ be a power-w-s, respectively, a power-s reduced words (11.30) in $\mathfrak{L} \mathcal{S}$. If there exists at least one $n_{l_{0}} \in\{\eta\}$, such that $n_{l_{0}}$ is odd in $\mathbb{N}$, then $X_{N}$ is 0 -close to $M_{N}$ in $\mathfrak{L} \mathfrak{S}$. i.e.,

$$
\begin{aligned}
& \exists n_{l_{0}} \in\{\eta\}, \text { s.t., it is odd, } \\
& \Rightarrow X_{N} \text { is } 0 \text {-close to } M_{N} \text { in } \mathfrak{L S} \text {. }
\end{aligned}
$$

Proof. Under hypothesis, the weight-ratio

$$
R_{N}=r_{W}^{J: \eta}[\mathcal{Q}: \Theta]=\left(r_{0}^{k}\right)_{k=1}^{\infty}
$$

becomes the zero $\mathbb{R}$-sequence by the above lemma, where $r_{0}$ is in the sense of (11.31). So,

$$
\lim _{k \rightarrow \infty}\left(\max \left\{r_{0}, r_{0}^{2}, \ldots, r_{0}^{k}\right\}\right)=\lim _{k \rightarrow \infty} 0=0,
$$

by the above lemma. Equivalently, it shows that $X_{N}$ is 0 -close to $M_{N}$ in $\mathfrak{L S}$.

By the above close-ness condition (11.32), we now focus on the cases where $\omega_{n_{l}}=1$, equivalently, $\eta$ is even 
in the sense that all entries $n_{l}$ are even in $\mathbb{N}$, for all $l=1, \ldots, N=|\eta|$. If $\eta$ is even, then the quantity

$$
r_{0}=\prod_{l=1}^{N} p_{l}^{n_{l}\left(j_{l}+1\right)} \neq 0 \text { in } \mathbb{R},
$$

and hence, the weight-ratio $R_{N}$,

$$
R_{N}=r_{W}^{J: \eta}[\mathcal{Q}: \Theta]=\left(r_{0}^{k}\right)_{k=1}^{\infty}
$$

is a $\mathbb{R}$-sequence without zero entries.

Definition 11.4. Let $X_{N}=X_{W}^{J: \eta}$ be a power-w-s reduced word, and hence, $M_{N}=M_{W}^{J: \eta}$, the corresponding power-s reduced word of (11.30) in $\mathfrak{L} \mathfrak{S}$, where $N=|\eta|>1$ in $\mathbb{N}$. Assume that $\eta$ is even. Then we call such operators $X_{N}$ and $M_{N}$, an even power-w-s reduced word, respectively, an even power-s reduced word in $\mathfrak{L} \mathfrak{S}$.

We obtain the following additional close-ness condition of $X_{N}$ and $M_{N}$ of (11.30) in the radial-Adelic probability space $\mathfrak{L} \mathfrak{S}$.

Theorem 11.11. Let $X_{N}$ and $M_{N}$ be an "even" power-w$s$ reduced word, respectively an "even" power-s reduced word in $\mathfrak{L S}$ in the sense of (11.30), and let

$$
t_{0}=\prod_{l=1}^{N} p_{l}^{n_{l}\left(j_{l}+1\right)} \text { in } \mathbb{R} \text {. }
$$

(11.33) If $0<t_{0}<1$ in $\mathbb{R}$, then $X_{N}$ is $t_{0}$-close to $M_{N}$ in $\mathfrak{L} \mathfrak{S}$.

(11.34) If $t_{0}>1$ in $\mathbb{R}$, then $X_{N}$ is not close to $M_{N}$ in $\mathfrak{L S}$.

Proof. Let $R_{N}=r_{W}^{J: \eta}[\mathcal{Q}: \Theta]$ be the weight-ratio of $X_{N}$ and $M_{N}$. Then, by (11.10), one obtains

$$
R_{N}\left(t_{0}^{k}\right)_{k=1}^{\infty} \text {, where } t_{0}=\prod_{l=1}^{N} p_{l}^{n_{l}\left(j_{l}+1\right)} \text { in } \mathbb{R} \text {, }
$$

by the even-ness of $\eta$. So, it is not difficult to check that: if $t_{0}=1$ in $\mathbb{R}$, then $R_{N}$ is the constant sequence having all its entries 1 , i.e.,

$$
R_{N}=(1,1,1,1, \ldots)
$$

Thus, one can get that

$$
\lim _{k \rightarrow \infty}\left(\max \left\{r_{0}, r_{0}^{2}, \ldots, r_{0}^{k}\right\}\right)=\lim _{k \rightarrow \infty} 1=1 .
$$

So, if $t_{0}=1$, then $X_{N}$ is 1-close to $M_{N}$ in $\mathfrak{L} \mathfrak{S}$.

Suppose now that $0<t_{0}<1$ in $\mathbb{R}$. Then the sequence $R_{N}$ is a monotonically strictly decreasing sequence. So,

$$
\lim _{k \rightarrow \infty}\left(\max \left\{r_{0}, r_{0}^{2}, \ldots, r_{0}^{k}\right\}\right)=\lim _{k \rightarrow \infty} t_{0}=t_{0} .
$$

Therefore, if $0<t_{0}<1$, then $X_{N}$ is $t_{0}$-close to $M_{N}$ in $\mathfrak{L S}$.

Therefore, the close-ness condition (11.33) holds.
Assume now that $t_{0}>1$ in $\mathbb{R}$. Then, the weight-ratio $R_{N}$ is a monotonically strictly increasing sequence, implying that

$$
\lim _{k \rightarrow \infty}\left(\max \left\{r_{0}, r_{0}^{2}, \ldots, r_{0}^{k}\right\}\right)=\lim _{k \rightarrow \infty} t_{0}^{k}=\infty
$$

It shows that $X_{N}$ is not close to $M_{N}$ in $\mathfrak{L} \mathfrak{S}$. Therefore, the close-ness condition (11.34) holds true.

In the rest of this section, we refine the above main results (11.33) and (11.34) more in detail.

Let $X_{N}$ and $M_{N}$ be an even power-w-s reduced word, respectively an even power-s reduced word in $\mathfrak{L} \mathfrak{S}$, in the sense of (11.30), and let

$$
R_{N}=r_{W}^{J: \eta}[\mathcal{Q}: \Theta]=\left(r_{0}^{k}\right)_{k=1}^{\infty}
$$

be the weight-ratio of $X_{N}$ and $M_{N}$, where

$$
t_{0}=\prod_{l=1}^{N} p_{l}^{n_{l}\left(j_{l}+1\right)} \text { in } \mathbb{R} .
$$

Now, let $J=\left(j_{1}, \ldots, j_{N}\right)$ be the integer-sequence of $X_{N}$ and $M_{N}$, with the corresponding subset $\{J\}$ of $\mathbb{Z}$. Now, decompose $\{J\}$ by

$$
\{J\}=\left\{J_{+}\right\} \sqcup\left\{J_{-}\right\},
$$

where

$$
\left\{J_{+}\right\}=\{j \in\{J\}: j>-1\}
$$

and

$$
\left\{J_{-}\right\}=\{j \in\{J\}: j \leq-1\} \text { in } \mathbb{Z},
$$

where $J_{+}$and $J_{-}$are the maximal sub-sequences of $J$ inducing $\left\{J_{+}\right\}$, respectively $\left\{J_{-}\right\}$.

For example, let $J=(6,-1,0,1,-1,2)$, with $\{J\}=\{-1,0,1,2,6\}$ in $\mathbb{Z}$. Then

$$
\left\{J_{+}\right\}=\{1,2,6\} \text {, and }\left\{J_{-}\right\}=\{-1,0\}
$$

with

$$
J_{+}=(6,1,2) \text { and } J_{-}=(-1,0,1) .
$$

Depending on $J_{+}$and $J_{-}$obtained from (11.36), let's decide the sub-sequences $W_{+}$and $W_{-}$of the prime-sequence $W=\left(p_{1}, \ldots, p_{N}\right)$, under the rule: $p_{l} \in W_{i}$, if and only if there exists $j_{l} \in J_{i}$, for all $i \in\{+,-\}$, for all $l=1, \ldots, N$. Similarly, determine the sub-sequences $\eta_{+}$and $\eta_{-}$of $\eta$, under similar manner.

Then the first entry $t_{0}$ of (11.35) in the weight-ratio $R_{N}$ of $X_{N}$ and $M_{N}$ can be re-written as a $\mathbb{R}$-quantity,

$$
t_{0}=t_{0}^{+} t_{0}^{-},
$$

with 


$$
t_{0}^{+}=\prod_{j_{l} \in J_{+}} p_{l}^{n_{l}\left(j_{l}+1\right)} \text {, and } t_{0}^{-}=\prod_{j_{l} \in J_{-}} p_{i}^{n_{i}\left(j_{i}+1\right)},
$$

as a positive real number in $\mathbb{R}$.

By the close-ness conditions (11.33) and (11.34), and by (11.37), we obtain the following refined main result.

Theorem 11.12. Let $X_{N}$ be an even power-w-s reduced word, and $M_{N}$, the corresponding even power-s reduced word in $\mathfrak{L S}$ as in (11.30), and let $R_{N}=\left(t_{0}^{k}\right)_{k=1}^{\infty}$ be the weight-ratio $r_{W}^{J: \eta}[\mathcal{Q}: \Theta]$ of $X_{N}$ and $M_{N}$, where $t_{0}=\prod_{l=1} p_{l}^{n_{l}\left(j_{l}+1\right)}$ is in the sense of (11.35) in $\mathbb{R}$. Suppose the quantity $t_{0}$ is factorized by

$$
t_{0}=t_{0}^{+} t_{0}^{-} \text {in } \mathbb{R}
$$

where

$$
t_{0}^{+}=\prod_{j_{l} \in J_{+}} p_{l}^{n_{l}\left(j_{l}+1\right)}, \text { and } t_{0}^{-}=\prod_{j_{l} \in J_{-}} p_{i}^{n_{i}\left(j_{i}+1\right)}
$$

are in the sense of (11.37), where $J_{+}$and $J_{-}$are the subsequences in the sense of (11.36). If

$$
\frac{1}{t_{0}^{-}} \geq t_{0}^{+} \text {in } \mathbb{N}
$$

Then $X_{N}$ is $t_{0}$-closed to $M_{N}$ in $\mathfrak{L} \mathfrak{S}$.

If the above condition (11.38) does not hold, then $X_{N}$ is not close to $M_{N}$ in $\mathfrak{L} \mathfrak{S}$. Proof. Suppose $t_{0}=t_{0}^{+} t_{0}^{-}$in $\mathbb{R}$, as in (11.37). Then, by the very constructions,

$$
t_{0}^{+} \in \mathbb{N} \text {, and } \frac{1}{t_{0}^{-}} \in \mathbb{N} \text {, in } \mathbb{R} \text {. }
$$

Assume now the condition (11.38) holds in $\mathbb{N}$. Then the positive $\mathbb{R}$-quantity $t_{0}$ satisfies

$$
0<t_{0} \leq 1 \text { in } \mathbb{R} \text {. }
$$

Therefore, by (11.33), $X_{N}$ is $t_{0}$-close to $M_{N}$ in $\mathfrak{L S}$.

By (11.34), if the condition (11.38) does not hold, then $X_{N}$ is not close to $M_{N}$ in $\mathfrak{L} \mathfrak{S}$.

So, by (11.32), (11.33), (11.34), and (11.38), one can summarize the close-ness of power-w-s reduced words as follows.

Corollary 11.13. Let $X_{N}$ and $M_{N}$ be a power-w-s reduced word, respectively, a power-s reduced word in $\mathfrak{L S}$, and let

$$
R_{N}=\left(r_{0}^{k}\right)_{k=1}^{\infty}=r_{W}^{J: \eta}[\mathcal{Q}: \Theta]
$$

with

$$
r_{0}=\prod_{l=1}^{N} \omega_{n_{l}} p_{l}^{n_{l}\left(j_{l}+1\right)} \text { in } \mathbb{R}
$$

(I) If there exists at least one $n_{l} \in\{\eta\}$, such that $n_{l}$ is odd in $\mathbb{N}$, then $X_{N}$ is 0 -close to $M_{N}$ in $\mathfrak{L} \mathfrak{S}$.

(II) Let $X_{N}$ and $M_{N}$ be an even power-w-s, respectively an even power-s reduced words in $\mathfrak{L} \mathfrak{S}$, and let $r_{0}=t_{0}^{+} t_{0}^{-}$be decomposed by (11.37). If $\frac{1}{t_{0}^{-}} \geq t_{0}^{+}$in $\mathbb{N}$, then $X_{N}$ is $r_{0}$-close to $M_{N}$ in $\mathfrak{L} \mathfrak{S}$. Otherwise, $X_{N}$ is not close to $M_{N}$ in $\mathfrak{L S}$.

The above corollary provides the close-ness of power-W-s reduced words and power-s reduced words in $\mathfrak{L S}$.

By the above close-ness, and by the identically-freedistributedness, one obtains the following corollary, too.

Corollary 11.14. Let $X=X_{W}^{J: \eta}$ be a power-w-s reduced word of (11.30) in the radial-Adelic probability space $\mathfrak{L S}$, and let $S$ be a free reduced word $S=\prod_{l=1}^{N} S_{l}^{n_{l}}$ in an arbitrary topological *-probability space $(A, \varphi)$ in $(A, \varphi)$, where $\left(s_{1}, \ldots, s_{N}\right)$ is an alternating $N$-tuple of "free," semicircular elements of $(A, \varphi)$, and where $s_{N} \neq s_{1}$ in $A$, for $N>1$ in $\mathbb{N}$. Let

$$
r(X, S)=\left(\frac{\tau^{0}\left(X^{(k)}\right)}{\varphi\left(S^{(k)}\right)}\right)_{k=1}^{\infty}=\left(r_{k}\right)_{k=1}^{\infty}
$$

be a $\mathbb{R}$-sequence. Then there exists a nonnegative number $r_{0}$ in $\mathbb{R}$, such that

$$
r_{0}=\prod_{l=1}^{N} \omega_{n_{l}} p_{l}^{n_{l}\left(j_{l}+1\right)}, \text { and } r(X, S)=\left(r_{0}^{k}\right)_{k=1}^{\infty} .
$$

Moreover, we have the following cases:

(11.39) If there exists at least one $n_{l} \in\{\eta\}$, such that $n_{l}$ is odd in $\mathbb{N}$, then

$$
\lim _{k \rightarrow \infty} r_{0}^{k}=0
$$

(11.40) If $X$ is an even power-w-s reduced word in $\mathfrak{L S}$, then $r_{0}>0$ in $\mathbb{R}$, and

$$
\lim _{k \rightarrow \infty} r_{0}^{k}= \begin{cases}r_{0} & \text { if } 0<r_{0} \leq 1 \\ 0 & \text { otherwise }\end{cases}
$$

Proof. The proofs of (11.39) and (11.40) are done by the identically-free-distributedness of our power-s reduced word $M_{N}$ of (11.30) and the free reduced word $S$, with help of (11.32), (11.33), (11.34) and (11.38).

The above corollary shows the close-ness between free distributions of our power-w-s reduced words of the radial-Adelic probability space $\mathfrak{L S}$ generated by the free weighted-semicircular family $\mathcal{Q}$, and free distributions of certain free reduced words generated by an arbitrary free semicircular family. 


\section{References}

[1] I. Cho, Representations and Corresponding Operators Induced by Hecke Algebras, Compl. Anal. Oper. Theo., 10, no. 7, (2016). 1453-1499.

[2] I. Cho, Free $W^{*}$-Dynamical Systems from $p$-Adic Number Fields and the Euler Totient Functions, Mathematics, 3, (2015). 1095-1138.

[3] I. Cho, On Dynamical Systems Induced by p-Adic Number Fields, Opuscula Math., 35, no. 4, (2015). 445-484.

[4] I. Cho, Free Semicircular Families in Free Product Banach *-Algebras Induced by $p$-Adic Number Fields over Prims $p$, Compl. Anal. Oper. Theo., 11, no. 3, (2017). 507-565.

[5] I. Cho, Free Distributional Data of Arithmetic Functions and Corresponding Generating Functions, Compl. Anal. Oper. Theo., vol. 8, issue:2, (2014). 537-570.

[6] I. Cho, Dynamical Systems on Arithmetic Functions Determined by Prims, Banach J. Math. Anal., 9, no. 1, (2015). 173-215.

[7] I. Cho, and T. Gillespie, Free Probability on the Hecke Algebra, Compl. Anal.Oper. Theo., vol. 9, issue 7, (2015). 1491-1531.
[8] I. Cho, and P. E. T. Jorgensen, Krein-Space Operators Induced by Dirichlet Char-acters, Special Issues: Contemp. Math.: Commutative and Noncommutative Harmonic Analysis and Applications, Amer. Math. Soc., (2014). 3-33.

[9] T. Gillespie, Superposition of Zeroes of Automorphic L-Functions and Functoriality, Univ. of Iowa, PhD Thesis. (2010).

[10] T. Gillespie, Prime Number Theorems for Rankin-Selberg $L$-Functions over Number Fields, Sci. China Math., 54, no. 1, (2011). 35-46.

[11] F. Radulescu, Random Matrices, Amalgamated Free Products and Subfactors of the $C^{*}$-Algebra of a Free Group of Nonsingular Index, Invent. Math., 115, (1994). 347-389.

[12] R. Speicher, Combinatorial Theory of the Free Product with Amalgamation and Operator-Valued Free Probability Theory, Amer. Math. Soc. Mem., vol 132, no. 627, (1998).

[13] V. S. Vladimirov, I. V. Volovich, and E. I. Zelenov, p-Adic Analysis and Math-ematical Physics, Ser. Soviet \& East European Math., vol 1, (1994). World Scientific.

[14] D. Voiculescu, K. Dykemma, and A. Nica, Free Random Variables, CRM Monograph Series, vol 1., (1992). Published by Amer. Math. Soc.. 